\title{
Tabularia
}

TABULARIA Sources écrites des mondes normands médiévaux Jumièges, foyer de production documentaire | 2007

\section{Vie perdue de Guillaume Longue-Épée († 942) état des recherches en cours}

The lost life of Guillaume "the long sword" (died in 942). The research as it is being carried out

Jacques Le Maho

\section{CpenEdition}

Journals

Édition électronique

URL : http://journals.openedition.org/tabularia/2075

DOI : $10.4000 /$ tabularia.2075

ISSN : 1630-7364

Éditeur :

CRAHAM - Centre Michel de Boüard, Presses universitaires de Caen

Référence électronique

Jacques Le Maho, «Vie perdue de Guillaume Longue-Épée († 942) état des recherches en cours », Tabularia [En ligne], Jumièges, foyer de production documentaire, mis en ligne le 11 septembre 2007, consulté le 19 avril 2019. URL : http://journals.openedition.org/tabularia/2075 ; DOI : 10.4000/ tabularia.2075 


\title{
Vie perdue de Guillaume Longue-Épée († 942), état des recherches en cours
}

\section{The lost life of Guillaume "the long sword" (died in 942). The research as it is being carried out}

\author{
Jacques LE MAHO (CNRS) \\ CRAHM-UMR 6577 \\ Université de Caen Basse-Normandie \\ jacques.lemaho@unicaen.fr
}

Résumé:

L'analyse comparative de plusieurs textes des $\mathrm{X}^{\mathrm{e}}$ et $\mathrm{XI}^{\mathrm{e}}$ siècles, dont le Planctus sur la mort de Guillaume Longue-Épée, les livres II et III de l'ouvrage de Dudon de Saint-Quentin, l'Histoire de France de Richer de Reims et les Gesta de Guillaume de Jumièges, amène à la constatation que ces textes dérivent probablement tous, à des degrés divers, d'une même source narrative aujourd'hui perdue, une Vie de Guillaume Longue-Épée, deuxième duc de Normandie (v. 927-942). Sans doute réalisée au monastère de Saint-Mesmin-de-Micy (Loiret) à une date comprise entre 950 environ et 963, cette œuvre est attribuable à Annon, abbé de Micy et de Jumièges ( $†$ 970/973), également identifié depuis quelques années comme l'auteur des Vies des saints Aycadre et Hugues de Jumièges ainsi que de la Vie de saint Eucher d'Orléans. Les recherches en cours portent notamment sur un essai de reconstitution du synopsis de la Vita et sur la genèse des livres II et III de Dudon, ceux-ci se révélant aujourd'hui, non plus comme le texte fondateur de l'historiographie des deux premiers ducs, mais comme une simple réécriture de la Vita Guillelmi d'Annon.

Mots-clés: Annon, biographie, complainte, Gerbert d'Aurillac, Guillaume de Jumièges, Guillaume Longue-Épée, Jumièges, Orléans, Reims, Richard I ${ }^{\text {er }}$, Richer, Rouen, Rollon, Saint-Mesmin de Micy, vita.

\begin{abstract}
:
The comparative analysis of several texts dating back from the $10^{\text {th }}$ and $11^{\text {th }}$ centuries, among which the Planctus on the death of Guillaume the long sword, the volumes II and III of the work by Dudon of St Quentin, the History of France by Richer of Reims and the Gesta of Jumièges, brings to the establishment that all these texts, in various degrees, probably derive from one narrative source - lost now -: A life of Guillaume the long sword, second earl of Normandy (927-942). Most certainly written in the monastery of St Mesmin of Micy (Loiret), some time between 950 and 963, this work is to be ascribed to Annon, abbot of Micy and Jumièges (died in 970/973), a man who, for some years, has also been identified as the author of the lives of saint Aycadre and saint Hugues of Jumièges as well as of the life of saint Eucher of Orleans. The research which is being currently carried out notably deals with an attempt at rebuilding the synopsis of the Vita and with the origins of the volumes II and III by Dudon, these books being acknowledged now as a simple rewriting of the Vita Guillelmi by Annon, and no longer as the founding text of the historiography of the first two earls.
\end{abstract}


Keywords: Annon, biography, lament, Gerbert d'Aurillac, Guillaume de Jumièges, Guillaume Longue-Épée, Jumièges, Orléans, Reims, Richard I, Richer, Rouen, Rollon, Saint-Mesmin de Micy, Vita.

En 2001, à la fin d'une étude relative à deux textes hagiographiques du $\mathrm{X}^{\mathrm{e}}$ siècle écrits pour l'abbaye de Jumièges ${ }^{1}$, nous avons annoncé une suite. Elle sera consacrée à une œuvre perdue qui a dû constituer une des pièces principales du corpus de sources narratives de Jumièges, mais qui nous est également apparue, au fur et à mesure de l'avancement de nos recherches, comme une source majeure de l'histoire du duché: une Vie de Guillaume Longue-Épée, deuxième duc de Normandie et restaurateur de l'abbaye de Jumièges, mort en 942.

Cinq années s'étant déjà écoulées depuis la parution de l'article de Tabularia, nous devons présenter nos excuses pour le retard de la publication annoncée. Il est clair aujourd'hui que nous n'avions pas bien mesuré, lors de nos premières investigations, l'ampleur du nouveau champ de recherches auquel elles ouvraient la porte. Sans plus attendre, il nous a cependant semblé que le moment était venu de livrer ces quelques informations sur l'avancement de nos travaux.

\section{La Vita prima Guillelmi}

L'enquête trouve son point de départ dans un certain nombre de réflexions auxquelles nous avaient conduit nos recherches textuelles à partir du milieu des années 1990. À cette date, nous avions acquis la conviction que la Complainte sur la mort de Guillaume Longue-Épée, chant funèbre composé peu après la mort du duc en 942, n'était pas une œuvre tout-à-fait originale ${ }^{2}$. En raison de sa structure condensée, de sa formulation souvent elliptique et d'un plan qui - particularité peu fréquente pour un planctus de cette époque - semble suivre le déroulement chronologique d'une narration, il nous apparaissait qu'elle était sans doute le résumé, sous une forme rimée, d'une biographie en prose, de la même façon qu'un office ou une hymne composée en l'honneur d'un saint procède d'un résumé de sa Vita ${ }^{3}$. D’où l'hypothèse d'une Vita primitive de Guillaume, non

1. Le Maнo, Jacques, «La production éditoriale à Jumièges vers le milieu du $\mathrm{X}^{\mathrm{e}}$ siècle» (première partie), Tabularia «Études», n 1, p. 11-32, 22 octobre 2001.

2. Complainte de Guillaume Longue-Épée, éd. Jules LaIr, Étude sur la vie et la mort de Guillaume Longue-Épée, Paris, Picard, 1893, p. 61-68; LAuer, Philippe, Le règne de Louis IV d'Outremer, Paris, Champion, 1900, p. 319-323; BECKer, Phillipp August, «Der Planctus auf den Normannenherzog Wilhelm Langschwert (942)», in Zeitschrift für französische Sprache und Literatur (63, 1939), p. 190-197; MEYER, Wilhelm, Gesammelte Abhandlungen zur mittellateinischen Rythmik, Berlin, 1905, tome 1, p. 218-219 (Weidmannsche Buchhandlung). Nous utilisons ci-après l'édition de Philippe Lauer [désormais Complainte]. Olivier Diard prépare actuellement une étude sur le Planctus de Guillaume en tant qu'hymne (avec la collaboration de Pascal Pradié et de nousmême sur les aspects textuels).

3. Sur ce type de transformation textuelle, voir les précieuses remarques de Jean-Yves TiLliETtE, «Hymnes et séquences hagiographiques: formes et fonctions de la réécriture lyrique des vies 
seulement antérieure à celle écrite par Dudon de Saint-Quentin aux environs de l'an Mille (livre III de l'édition de Jules Lair), mais également au Planctus luimême.

Il y avait plusieurs motifs pour penser que le Planctus n'était pas la seule œuvre dérivée de la Vita perdue. Les récits que Richer de Reims (v. 990-994) et Dudon de Saint-Quentin nous livrent de l'assassinat de Guillaume Longue-Épée sont si détaillés et présentent tant de points de concordance que les historiens ont été nombreux, depuis la fin du XIX ${ }^{\mathrm{e}}$ siècle, à soupçonner l'existence d'une source commune, tour à tour utilisée par Richer et Dudon ${ }^{4}$. Certains ont pensé à une tradition orale qui se serait transmise à travers un chant populaire ou un récit épique, ce qui est une réponse logique, mais sans doute un peu trop commode, au problème de l'absence de trace écrite ${ }^{5}$. Sachant que plusieurs strophes du Planctus sont également consacrées au récit de l'assassinat de Guillaume, d'autres ont vu dans cette complainte funèbre la source originelle ${ }^{6}$. Toutefois, comme il arrive souvent lorsqu'un texte en prose a été remanié et condensé pour être retranscrit en vers, la formulation en est si allusive et parfois si obscure que l'on voit mal comment Richer et Dudon auraient pu chacun de leur côté, à partir de ce seul texte, reconstituer deux récits aussi complets et aussi convergents ${ }^{7}$. Il en est de même pour plusieurs autres épisodes de la vie de Guillaume. Lorsque le duc rencontre un jour l'abbé Martin à Jumièges, les propos du second se réduisent dans le Planctus à une formule sibylline sur le mystère de la Sainte Trinité $^{8}$. Il est peu probable que ce passage énigmatique, difficilement compréhensible si l'on ignore le contexte, ait suffi à Dudon pour en déduire que le discours de Martin était axé sur la théorie de la tripartition sociale, que cette argumentation constituait la réponse à une question posée par le duc et que ce dernier

de saints», Hagiographica, Florence, Società internationale per lo studio del medioevo latino, 1994, p. 161-181.

4. Richer von Saint-Remi, Historiae, éd. Hartmut Hoffmann, Monumenta Germaniae Historica, Scriptores, t. XXXVIII, Hanovre, Hahn, 2000 [désormais RICHER], p. 121-122; DUdon DE SAINTQuentin, De gestis Normanniae ducum seu de moribus et actis primorum Normanniae ducum, éd. Jules LaIr, Mémoires de la Société des Antiquaires de Normandie, t. XXIII, Caen, Société des antiquaires de Normandie, 1865 [désormais Dudon], p. 205-208. Il est aujourd'hui à peu près certain que le manuscrit de Richer resta à l'état d'ébauche et ne fut jamais diffusé. L'hypothèse d'emprunts de Dudon à cette œuvre est donc à exclure.

5. Lauer, Philippe, Le règne de Louis IV..., p. 267-276; pour Robert Latouche, ce passage de l'œuvre de Richer procède de «traditions légendaires » (RICHER, Histoire de France (888-995), éd. Robert Latouche, Les Classiques de l'Histoire de France au Moyen Âge, Paris, Honoré Champion, 1930, t. 1, p. 171, n. 3 ).

6. Prentout, Henri, Étude critique sur Dudon de Saint-Quentin et son Histoire des premiers ducs normands, Paris, Picard, 1916, p. 304 et suiv. Cette interprétation a encore aujourd'hui la faveur de nombreux spécialistes. Voir par exemple Albu, Emily, The Normans in their Histories: Propaganda, Myth and Subversion, Woodbridge, The Boydell Press, 2001, p. 64; BARThÉLÉmy, Dominique, Chevaliers et miracles, La violence et le sacré dans la société féodale, Paris, Armand Colin (Collection Les enjeux de l'histoire), 2004, p. 32-33.

7. Voir à la fin du présent article, en annexe III, la liste des principales concordances entre les récits des deux auteurs.

8. «Idem doctus Trinitatis unitatem/a Martino, unitatis Trinitatem,/tria unum atque unum tria esse» (Complainte, $\mathrm{V})$. 
s'était confié à l'abbé de Jumièges parce qu'il était personnellement en proie à un doute eschatologique: avait-il de meilleures chances de salut s'il quittait sa condition de laïque - ce qui impliquait l'abandon du pouvoir - pour rejoindre l'ordre des moines? Lorsqu'il rédigea ce chapitre-clé de la Vita Guillelmi, il est donc assez évident que Dudon avait un texte sous les yeux et que celui-ci présentait une version beaucoup plus développée que le Planctus. Enfin, comme cela a été montré par plusieurs auteurs et notamment, en dernier lieu, par Pierre Bouet, la Vita Guillelmi de Dudon s'apparente très nettement, dans sa thématique comme dans la forme du discours, à une vie de saint ${ }^{9}$. Notre interprétation diffère cependant de celle de Pierre Bouet en ce sens que cette particularité du livre III du De moribus ne nous paraît pas résulter d'un choix délibéré de Dudon, mais bien plutôt, en l'occurrence, de la nature de sa source. Dans sa biographie de Richard I ${ }^{\text {er }}$, non moins laudative, mais davantage basée sur les écrits de Flodoard (pour les premières années du principat) et sur des témoignages oraux ou des souvenirs personnels, la thématique, les codes narratifs et même le lexique sont différents.

De même que bon nombre de récits hagiographiques ne sont que la refonte d'une Vita antérieure, nous sommes ainsi parvenu à la conclusion que la Vita Guillelmi constituant le livre III de l'ouvrage de Dudon n'est pas, elle non plus, une composition originale, mais le produit de la réécriture d'une Vita plus ancienne, et que cette dernière a également de bonnes chances de constituer la source de certains passages du livre II relatifs à l'histoire de Rollon, père de Guillaume. Ces observations ne s'appliquent pas uniquement à l'œuvre de Dudon. De nombreux recoupements donnent à penser que le Planctus sur la mort de Guillaume, mais aussi une grande partie des chapitres de l'Histoire de Richer concernant le duc normand (siège de Montreuil de 939, relations de Guillaume avec le roi Louis IV d'Outremer, réunion de Visé/Attigny et assassinat de Picquigny), procèdent de la même Vita perdue ${ }^{10}$. À ces textes dérivés, il convient d'ajouter les premières Annales de Rouen (avant 1087) qui recèlent plusieurs informations originales sur Guillaume Longue-Épée, les Gesta de Guillaume de Jumièges, où l'on trouve un récit détaillé de la restauration de Jumièges en 942, et enfin les annales de cette même abbaye, qui mentionnent plusieurs autres faits inédits relatifs à cette restauration ${ }^{11}$. En l'état actuel de nos recherches, les textes formant la descendance de la Vita prima Guillelmi semblent

9. Jordan, Victoria B., «The Role of Kingship in Tenth-Century Normandy: Hagiography of Dudo of Saint-Quentin», The Haskins Society Journal, 3, 1991, p. 53-63; BouEt, Pierre, «Dudon de SaintQuentin et le martyre de Guillaume Longue-Épée», in Les Saints dans la Normandie médiévale, Actes du colloque de Cerisy-la-Salle (26-29 septembre 1996), Pierre Bouet et François Neveux (éd.), Caen, Presses Universitaires de Caen, 2000, p. 220-243.

10. RicheR, p. 107-108, 112, 119-122.

11. Chronicum Rotomagense, éd. Philippe LabBe, Novae Bibliothecae manuscriptorum librorum, t. 1, Paris, 1657, p. 364-366; The Gesta Normannorum Ducum of William of Jumièges, Orderic Vitalis and Robert of Torigni, éd. et trad. Elisabeth VAN Houts, Oxford, Clarendon Press (Oxford Medieval Texts) [désormais GND], t. 1, 1992, p. 18-20 et 84-86; Les Annales de l'abbaye Saint-Pierre de Jumièges, chronique universelle des origines au XIII siècle, éd. et trad. dom Jean LAPORTE, Rouen, Lecerf, 1954, p. 78-84. 
donc se répartir en deux groupes, d'une part ceux qui relèvent d'un projet global de réécriture (Planctus sur la mort de Guillaume, Vita Guillelmi de Dudon), d'autre part ceux où la Vita n'a été utilisée que de manière partielle ou ponctuelle, comme source d'informations annalistiques ou historiographiques (Histoire de Richer, Gesta de Guillaume de Jumièges, Annales de Rouen, Annales de Jumièges).

Sauf dans l'hypothèse, hélas assez improbable, de la découverte d'un manuscrit oublié, il va de soi que l'on ne peut espérer pouvoir reconstituer autre chose que quelques fragments du texte original. Toutefois, les œuvres dérivées nous livrent assez d'éléments pour parvenir à une idée relativement précise du contenu de la Vie perdue.

Pour cette partie de l'enquête, la base méthodologique nous a été fournie par les importants travaux de Monique Goullet sur la réécriture hagiographique ${ }^{12}$. Les recherches de Joseph-Claude Poulin sur les Vies de saint Samson, avec son essai de reconstitution du synopsis de la Vita prima perdue, ont également été pour nous un exemple très stimulant ${ }^{13}$. Dans une telle entreprise, toute la difficulté consiste à faire le partage entre les apports personnels des remanieurs (additions, réductions, corrections, permutations, transformations stylistiques, etc.) et les éléments susceptibles de provenir de l'oeuvre primitive. En effet, ce travail de décryptage n'est possible que si l'on est parvenu à une connaissance intime du projet et du mode d'écriture de chaque réutilisateur de la source originale.

Chez Richer, il semble que la Vita Guillelmi ne soit là que pour apporter un certain nombre de compléments à sa source de base, les Annales de Flodoard. Ces interpolations du jeune élève de Gerbert d'Aurillac, et c'est une chance pour nous car elles sont d'autant plus faciles à repérer, sont effectuées de façon particulièrement maladroite. Lorsqu'il évoque la prestation de serment de Guillaume au roi Louis IV et qu'il présente à cette occasion le duc de Normandie comme un modèle absolu de fidélité, de loyauté et d'esprit de sacrifice, Richer n'a visiblement pas mesuré combien ce passage - inspiré, selon toute apparence, de la Vita Guillelmi - s'accorde mal avec le portrait qu'il donne ailleurs du même Guillaume d'après les Annales de Flodoard, celui d'un «duc des pirates» brutal et sans parole, à l'affût de la moindre occasion d'envoyer ses bandes de pillards dévaster le royaume ${ }^{14}$. De même, lorsqu'il évoque les préparatifs du guet-apens

12. Goullet, Monique, "Vers une typologie des réécritures hagiographiques...", in La réécriture hagiographique dans l'Occident médiéval, transformations formelles et idéologiques, Monique Goullet et Martin Heinzelmann (dir.), Beihefte der Francia, 58, 2003, p. 109-144; ID., Écriture et réécriture hagiographiques, essai sur les réécritures de Vies de saints dans l'Occident latin médiéval, VIII ${ }^{\mathrm{e}}$-XIII ${ }^{\mathrm{e}}$ s., Turnhout, Brepols, 2005 (Hagiologia 4), $318 \mathrm{p}$.

13. Poulin, Joseph-Claude, «La "Vie ancienne" de saint Samson de Dol comme réécriture $(B H L$ 7478-7479)", Analecta Bollandiana, t. 119, II - décembre 2001, p. 261-312.

14. "Unde et regis factus, tanto ei consensu alligatus est ut iam iamque aut sese moriturum, aut regi imperii summa restiturum proponeret » (RICHER, II, 20). Cette phrase a suscité maints commentaires chez les historiens. Elisabeth NorTiER en fait une lecture au premier degré, y voyant le témoignage d'un attachement et d'un dévouement réels de Guillaume de Normandie à l'égard du roi («La féodalité en crise. Propos sur «Fiefs and Vassals» de Susan Reynolds», Revue historique, CCXCVI/2, 1997, p. 326). Pour Dominique BARThéLÉmy, ce ne peut être qu'une invention 
tendu contre Guillaume à la fin de l'année 942, Richer raconte par avance le scénario complet de l'assassinat (I. II, 32), ce qui, au risque d'ôter beaucoup de son intérêt à la narration, l'amène à se répéter lorsqu'il passe au récit des faits (l. II, 33). Ce genre de redite est fréquent chez les chroniqueurs en manque de sources, le procédé le plus simple pour dissimuler une lacune documentaire étant de décliner sous différentes formes ou sous différents angles narratifs, au fur et à mesure du déroulement du récit, les mêmes informations. Enfin, pour lever les derniers doutes sur la source des interpolations de Richer, reportons-nous, encore une fois, au récit de l'assassinat de Guillaume. Le manuscrit autographe de Bamberg présente, à cet endroit, un léger remaniement: au mot exspectantem indiquant que l'assassin a prévu d'attendre sa victime de l'autre côté de la rivière, Richer a ajouté après coup le mot prestolantem ${ }^{15}$. Cette addition n'a aucune justification apparente, mais elle prend tout son sens quand on la rapproche du passage correspondant de la Complainte où, dans les mêmes termes curieusement redondants, il est précisé que le comte Arnoul attend sur la rive le retour de Guillaume: expectante prestolando ${ }^{16}$. C'est bien la preuve que Richer a connu et utilisé la Vie de Guillaume Longue-Épée, soit dans la version brève que constitue le chant de la Complainte, soit plutôt, puisque tout laisse supposer que Richer a eu accès à une narration beaucoup plus détaillée, dans la version originale en prose, aujourd'hui perdue.

Chez Guillaume de Jumièges, l'objectif est clairement affiché dans le prologue: dans les premiers chapitres de ses Gesta, l'auteur se bornera à fournir un résumé de l'œuvre de Dudon. Il n'a donc recours à la Vita Guillelmi que pour préciser certains détails omis par le chanoine ou pour réintroduire les développements que celui-ci n'avait pas jugé utile d'insérer dans sa geste des ducs; c'est notamment le cas pour les chapitres relatifs à Jumièges, aux origines de l'abbaye et à sa restauration sous l'égide de Guillaume Longue-Épée. À ces exceptions près, il semble néanmoins que la version de Dudon de Saint-Quentin nous livre un reflet assez complet du contenu de l'oeuvre primitive. Pour autant, la recherche des traces de la Vita prima est loin d'être facile chez Dudon. Non seulement l'hypotexte a été l'objet, de la part de cet auteur doté d'une forte personnalité littéraire, d'importantes amplifications et d'une refonte complète sur le plan formel ${ }^{17}$, mais de surcroît, il est certain que Dudon en a plus d'une fois modifié

de Richer (Chevaliers et miracles..., p. 32-33). Mention de bandes de vikings qui, à l'appel de Guillaume, débarquent en France et dévastent l'Aquitaine: Richer, I, 57. Mentions de Guillaume «dux piratarum», "princeps pyratarum»: ibid., II, 20, 28, 30.

15. «ad dominum cum copiis exspectantem (prestolantem)» (RICHER, II, 33, p. 122).

16. «Hoc cis intuens expectante prestolando " (Complainte, XII, p. 322).

17. Sur les aspects stylistiques de l'œuvre de Dudon, voir en particulier: Sноркоw, Leah, «The Carolingian world of Dudo of Saint-Quentin», Journal of Medieval History, 1989, $\mathrm{n}^{\circ}$ 15, p. 1937; Bouet, Pierre, «Dudon de Saint-Quentin et Virgile: l'«Enéide» au service de la cause normande", in Recueil d'études en hommage à Lucien Musset, Cahiers des Annales de Normandie, $\mathrm{n}^{\circ} 23$, Caen, 1990, p. 215-236; AlBu, Emily, The Normans in their Histories, p. 7-46; LeCOUTEUX, Stéphane, "À partir de la diffusion de trois poèmes hagiographiques, identification des centres carolingiens traditionnels ayant influencé l'œuvre de Dudon de Saint-Quentin», Tabularia «Études», $\mathrm{n}^{\circ} 5,2005$, p. 13-49, 26 avril 2005. 
l'ordonnance et le contenu, déplaçant ici un épisode pour le réutiliser ailleurs, substituant tel personnage à tel autre, voire même, sur des sujets politiquement sensibles comme le lieu de naissance de Guillaume Longue-Épée, imposant une version radicalement différente de celle d'Annon ${ }^{18}$. Pour savoir quels passages du récit de Dudon procèdent d'emprunts à l'œuvre originale, nous avons donc dû nous familiariser d'abord avec sa méthode, identifier ses autres sources, ses codes narratifs et ses thèmes de prédilection, réexaminer chaque épisode en fonction du contexte politique et culturel des environs de l'an Mille. Bien que la voie ait été déjà largement ouverte en ce domaine par Henri Prentout dès 1916 et que les travaux récents d'Éric Christiansen aient permis de beaucoup progresser dans le décryptage du discours du chanoine ${ }^{19}$, ce travail d'analyse a nécessité un élargissement considérable de notre champ de recherche.

Si nos investigations sont donc loin d'être terminées, il nous semble néanmoins que la structure générale de la Vita prima Guillelmi commence à se dégager. Vraisemblablement précédée d'une épitre dédicatoire adressée à Richard I ${ }^{\text {er }}$ († 996), fils et successeur de Guillaume Longue-Épée, et au comte de Poitiers Guillaume Tête d'Étoupe († 963), beau-frère du duc disparu (Planctus), puis d'un prologue sur le thème de la valeur rédemptrice du martyre (Planctus + Dudon), l'œuvre était sans doute composée de trois parties. D'après les recoupements entre le Planctus et les cinq autres textes dérivés, la première devait être consacrée aux origines de Guillaume (ses parents, sa naissance), à son portrait moral et à son œuvre politique (sa lutte victorieuse contre les rebelles, ses alliances, son soutien indéfectible au roi Louis IV), la deuxième aux œuvres pieuses de Guillaume, à sa quête spirituelle et à son attirance pour la vie monastique (refondation de l'abbaye de Jumièges, entretien avec l'abbé Martin), la troisième au complot ourdi contre lui par Arnoul de Flandre et à son assassinat à Picquigny.

Ainsi reconstitué dans ses grandes lignes, le plan de la Vita Guillelmi ressemble beaucoup à celui des trois livres de la Vie de Géraud d'Aurillac, écrite vers 930 par Odon, abbé de Cluny ${ }^{20}$. Cette similitude n'est sûrement pas le fait du hasard. L'influence de la Vita Geraldi est perceptible dans les passages du Planctus et de la Vita Guillelmi de Dudon où le duc de Normandie est présenté non seulement comme un «saint laïque», défenseur des faibles et des opprimés, apôtre de la non-violence, amoureux de la paix et de la justice, mais aussi comme

18. D’après la Complainte, Guillaume naquit outre Manche («Hic in orbe transmarino natus », Complainte, II); selon Dudon, il serait né à Rouen («Rotomagensi urbe exstitit oriundus», Dudon, III, 36, p. 179).

19. Prentout, Henri, Étude critique sur Dudon de Saint-Quentin et son Histoire des premiers ducs normands, Paris, Picard, 1916; Dudo of St Quentin, History of the Normans, trad. Éric Christiansen, Woodbridge, The Boydell Press, 1998, 260 p. [désormais Dudon, trad. ChrisTIANSEN].

20. Vita Geraldi (BHL 3411), P. L., t. 133, col. 639-704. Ce texte célèbre a suscité un nombre considérable de travaux. Voir notamment, en dernier lieu, les études publiées dans l'ouvrage collectif Guerriers et moines, conversion et sainteté aristocratiques dans l'Occident médiéval (IX ${ }^{\mathrm{e}}$-XII ${ }^{\mathrm{e}}$ siècle), Michel Lauwers (éd.), Collection d’études médiévales de Nice vol. 4, Antibes, Éditions APDCA, 2002, $678 \mathrm{p}$. 
un des derniers fidèles du roi ${ }^{21}$. À l'instar de Géraud, qui dissimule sa tonsure sous une coiffe, Guillaume rêve depuis son adolescence de devenir moine, et il ira jusqu'à se faire remettre en secret un habit monastique. Au récit des luttes de Géraud contre les seigneurs brigands d'Auvergne répond celui des combats menés par Guillaume de Normandie contre les odieux agresseurs Rioulf et Arnoul, aux chapitres sur la fondation du monastère d'Aurillac font écho ceux consacrés dans la Vita Guillelmi à la restauration de l'abbaye de Jumièges. La filiation textuelle devient tout-à-fait évidente lorsque Dudon rapporte le contenu de l'entretien du duc avec l'abbé Martin, l'épisode offrant un parallèle exact avec celui de la Vita Geraldi où Odon nous fait les témoins d'une discussion entre Géraud et l'évêque de Cahors Gausbert: dans l'un et l'autre cas, l'homme d'Église s'emploie à convaincre le comte que son devoir est de rester auprès de ses sujets et, par conséquent, de renoncer à son projet d'entrer au cloître. Or, nous savons qu'en 934, soit huit ans avant son arrivée à Jumièges où il fut chargé par le duc Guillaume Longue-Épée de rétablir la vie monastique, l'abbé Martin avait reçu la mission de relever le monastère de Saint-Augustin de Limoges, que cette mission lui avait été confiée par l'évêque de Limoges Turpion et que ce dernier n'était autre que le commanditaire, avec son frère Aimon, abbé de Saint-Martial, de la Vie de Géraud d'Aurillac ${ }^{22}$. Selon toute vraisemblance, la Vita Geraldi était donc connue des moines de Saint-Cyprien de Poitiers qui, sous la conduite de l'abbé Martin, vinrent prendre possession de l'abbaye de Jumièges en 942. Écrite pour fournir un modèle de vie chrétienne aux princes, on peut penser que cette biographie avait été bien accueillie dans le monastère de Saint-Cyprien, devenu depuis peu le principal sanctuaire dynastique des comtes de Poitiers ${ }^{23}$.

\section{L'auteur}

L'une des conclusions de notre première étude était que la restauration monastique du $\mathrm{X}^{\mathrm{e}}$ siècle à Jumièges avait eu pour corollaire la rédaction de deux vies de saints consacrées à deux grandes figures de l'histoire de l'abbaye: saint

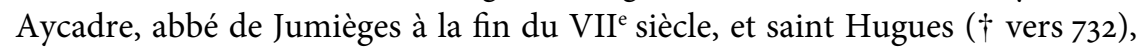
qui fut à la fois abbé de Jumièges et de Fontenelle, évêque de Rouen, de Bayeux et de Paris, mais que l'auteur croyait être un fils de Charlemagne, entré à Jumièges comme simple moine après avoir résigné sa charge d'archevêque (sic)

21. Sur ce dernier point, voir, pour la Vita Geraldi, Christian Lauranson-Rosaz, «La Vie de Géraud, vecteur d'une certaine conscience aristocratique dans le Midi de la Gaule», in Guerriers et moines..., p. 157-182.

22. BeCQuet, dom Jean, Actes des évêques de Limoges des origines à 1197, Documents, études et répertoires publiés par l'Institut de recherche et d'histoire des textes, 56, Paris, CNRS Éditions, 1999, $\mathrm{n}^{\circ}$, p. 26-27.

23. La précocité de la dévotion à saint Géraud en Poitou est confirmée par un calendrier de la première moitié du $\mathrm{X}^{\mathrm{e}}$ siècle; sur cet intéressant document, vraisemblablement d'origine poitevine, le nom de Géraud correspond à l'entrée la plus récente (Morin, Germain, «Un calendrier poitevin-breton du $\mathrm{X}^{\mathrm{e}}$ siècle», Jahrbuch fur Liturgiewissenschaft, t. 11, 1933, p. 78-93, en particulier p. 91-92). 
de Rouen ${ }^{24}$. De l'analyse textuelle, il résultait que cet auteur était vraisemblablement un abbé, peut-être Annon (943-970/973), successeur de Martin à Jumièges et en même temps, à partir du milieu du X $\mathrm{X}^{\mathrm{e}}$ siècle, abbé de Saint-Mesmin de Micy, près d'Orléans. S'il nous apparaît aujourd'hui qu'ils furent sans doute composés au scriptorium de Saint-Mesmin de Micy plutôt qu'à Jumièges comme nous l'avions d'abord supposé, en revanche de nouveaux éléments sont venu conforter l'attribution de ces textes à l'abbé Annon ${ }^{25}$. L'un d'eux réside dans le témoignage de Létald de Micy (fin du X ${ }^{e}$ siècle). En effet, le portrait qu'il nous a laissé de cet abbé correspond très exactement au profil de l'auteur des Vies de saint Aycadre et de saint Hugues: souci pointilleux du respect de la Règle de saint Benoît (c'est grâce à Annon, nous dit Létald, que fut rétablie l'observance bénédictine à Micy), primauté accordée aux vertus de l'hospitalité et de la charité, notamment à travers un usage dont l'abbé Annon avait fait un des principaux mots d'ordre à Micy et que l'on trouve évoqué de manière très répétitive tout au long des deux Vitae, l'offrande du repas ou du poculum caritatis (pas moins de 16 occurrences) ${ }^{26}$. En poursuivant nos recherches, il nous est également apparu que l'œuvre hagiographique d'Annon ne se résume sans doute pas aux Vies de saint Aycadre et de saint Hugues, divers recoupements permettant d'attribuer à ce même auteur la rédaction ou, plus vraisemblablement, la réécriture d'une Vie de saint Eucher, évêque d'Orléans (première moitié du VIII ${ }^{\mathrm{e}}$.) ${ }^{27}$.

Les œuvres hagiographiques d'Annon ne brillent guère par leur exactitude historique. Si, dans l'ensemble, la Vie de saint Eucher semble s'appuyer sur des sources relativement bien informées - ce qui s'explique dans l'hypothèse d'une simple réécriture, à partir d'une Vita primitive rédigée à l'abbaye de SaintTrond au diocèse de Liège, lieu d'inhumation d'Eucher -, en revanche les deux autres vies sont des œuvres de pure fiction, émaillées, comme nous venons de le constater pour la Vita Hugonis, d'erreurs grossières et d'anachronismes. Il ne faut cependant pas perdre de vue que le but essentiel d'Annon reste d'offrir à ses moines et à ses hôtes de Jumièges et de Micy, à travers une série d'exempla, différents modèles de sainte vie. En l'occurrence, le recours à la fiction lui permet de délivrer plus librement son message, sur un mode personnel et intimiste qui laisse deviner chez lui une longue expérience de confesseur et de guide spirituel: à chacun de savoir interpréter les signes que Dieu lui envoie pour lui fixer le cap à suivre dans son parcours terrestre. Malgré leurs graves défauts, les œuvres

24. De s. Aichardo abbate gemmeticensi in Normannia, Acta Sanctorum, 15 sept., sept. V, p. 80-102 (BHL 181); Van Der Straeten, Joseph, «Vie inédite de s. Hugues évêque de Rouen ", Analecta Bollandiana, 87, 1969, p. 215-26o (BHL 4032a). Désormais, les références à ces deux Vitae seront indiquées par les sigles $V A$ et $V H$, suivis du numéro du chapitre dans chacune des éditions.

25. Le MAно, Jacques, "Autour de la renaissance monastique du $\mathrm{X}^{\mathrm{e}}$ siècle en Normandie: les Vies des saints Aycadre et Hugues de Jumièges", in Livrets, collections et textes, études sur la tradition hagiographique latine, M. HeInZELmanN (dir.), Beihefte der Francia, 63, p. 285-322.

26. Ibid., p. 302-304

27. Ibid., p. 311-314 (Vita Eucherii Aurelianensis (BHL 2660), 9, Acta Sanctorum, Febr. III, p. 218 (I ${ }^{\text {ère }}$ éd.); éd. Bruno Krusch et Wilhelm Levison, MGH, Scriptores Rerum Merovingicarum, Hanovre et Leipzig, Hahn, VII, 1920, p. 41-53). Dorénavant, les renvois aux chapitres de la Vie de saint Eucher seront précédés des lettres $V E$. 
hagiographiques d'Annon sont donc loin d'être inintéressantes. Non seulement elles montrent les idées qu'un abbé du $\mathrm{X}^{\mathrm{e}}$ siècle pouvait se faire sur le monde, sur la société de son temps et sur les grands choix de l'existence, mais, de surcroît, cette richesse thématique permet des comparaisons très instructives avec les différentes questions abordées dans la Vita Guillelmi.

Pour Annon, de même qu'il existe une hiérarchie au sein du peuple céleste siégeant dans la maison de Dieu, la société humaine se divise en plusieurs groupes ou "ordres», différenciables par leur statuts canoniques. Ces groupes sont au nombre de trois: l'ordre des moines, celui des chanoines et celui des laïcs ${ }^{28}$. Au sein de cette classification tripartite, l'auteur ne cache pas qu'il accorde à l'ordo monastique une nette prééminence sur les deux autres. Parce qu'ils ont fait vœu de pauvreté et d'abstinence, mais aussi et surtout parce que leur condition les met à l'abri des turpitudes de ce monde, ce sont les moines qui se rapprochent le plus de Dieu et qui occuperont les meilleures places au sein du peuple des élus. Toutefois, de même que la Sainte Trinité est à la fois triple et indivisible, les trois groupes constitutifs du corps social ne doivent faire qu'un, dans une union sacrée, un esprit de concorde et de fraternité qui seront ici-bas le reflet de l'harmonie régnant au sein du peuple céleste. Concrètement, c'est par la pratique de la charité et de l'hospitalité, érigées au rang de vertus suprêmes, que sera maintenue cette nécessaire cohésion sociale. Là encore, les moines ont un rôle essentiel à jouer, non seulement par leur vocation d'accueil à l'égard des visiteurs et des hôtes de toutes conditions, mais aussi par leurs prières quotidiennes «pour la stabilité des trois ordres ${ }^{29}$.

Si ces idées sont rarement formulées de manière aussi explicite, en revanche elles sous-tendent en permanence le discours qu'Annon développe tout au long de ses récits hagiographiques. Dans la Vie de saint Aycadre, il retrace le parcours exemplaire d'un homme ayant fait très tôt le choix de la vie monastique. Sa dernière fonction est celle d'abbé de Jumièges, où il se montre le digne successeur de l'illustre saint Philibert. Les autres protagonistes du récit, qu'il s'agisse de l'évêque de Poitiers Ansoald (ordre clérical), des parents d'Aycadre ou d'un bienfaiteur de Jumièges (ordre laïque), sont tous cantonnés au rôle de serviteurs de la cause monastique. Avec la Vie de saint Hugues, soi-disant fils de Charlemagne, Annon se penche sur le cas d'un prince de très haut rang ayant, dès l'adolescence, secrètement décidé de se faire moine à Jumièges. Ce projet est contrarié par son père, qui le fait nommer archevêque de Rouen. Un beau jour, comprenant que cette fonction l'expose à de dangereuses compromissions avec le monde, Hugues décide de s'en affranchir en résignant sa charge épiscopale pour se retirer à l'abbaye de Jumièges, comme simple moine. Dernier volet du triptyque, la Vie de saint Eucher nous offre une autre variation sur le thème de la vocation monastique contrariée. Cette fois-ci, le héros de l'histoire est déjà

28. «ex omni ordine electos viros de plebibus, monachos videlicet in sancta religione famosos, nec non etiam canonicos, nec non etiam canonicos litterariae pertitiae eruditos atque laicos bone credulitatis ornatos » $(\mathrm{VH}, 29)$.

29. «... pro statu sanctae Dei ecclesiae et trium ordinum stabilitate » $(\mathrm{VH}, 27)$. 
moine à Jumièges lorsqu'on vient le chercher pour occuper la fonction d'évêque d'Orléans. Quitter l'ordre monastique pour rejoindre ainsi le monde est pour Eucher un vrai déchirement. Ce n'est qu'après avoir été exilé en Austrasie sur l'ordre de Charles Martel qu'il obtient l'autorisation de se retirer au monastère de Saint-Trond, où il pourra réaliser son vœu de finir ses jours sous l'habit monastique ${ }^{30}$. Tout au long de ces récits, Annon exalte à travers maintes descriptions de banquets, de réceptions et de cérémonies de bienvenue les vertus de la caritas et de l'hospitalitas. Ces évocations sont autant d'hymnes à la fraternité. Refuser l'offrande d'un repas, comme le fait Charles Martel en dédaignant les prandia préparés en son honneur par saint Eucher, est un geste de mauvais augure: il signifie que la concorde est rompue.

La correspondance de ces thèmes avec ceux de la Vita Guillelmi est tout à fait frappante. À Guillaume Longue-Épée qui l'interroge sur ses chances personnelles d'accéder au salut, l'abbé Martin répond par un discours où il formule une théorie de tripartition sociale fondée sur une classification identique à celle d'Annon: les moines, les chanoines et les laïcs sont les trois ordres constitutifs de la société ${ }^{31}$. De la relation passablement obscure que Dudon nous fait des propos de Martin, il ressort que, dans la version originale de la Vita Guillelmi, l'abbé devait établir un parallèle entre cette structure ternaire du corps social et le mystère de la Sainte Trinité, ce qui, là encore, correspond très exactement à la théorie qu'Annon expose dans ses œuvres hagiographiques. Le projet de Guillaume étant, toujours selon Dudon, de quitter sa condition de laïc pour rejoindre l'ordre des moines, il est probable que ceci formait le thème central de la Vita prima et que celle-ci insistait davantage sur la supériorité des vertus inhérentes à l'état monastique. Sur ce dernier point, la narration de Dudon laisse deviner parfois chez lui une certaine réserve, ce qui peut se comprendre quand on se souvient qu'il était chanoine de son état; rappelons également qu'il se présente comme un disciple d'Adalbéron de Laon, auteur de violents pamphlets contre ces moines clunisiens qui, pour reprendre les termes de Georges Duby, prétendaient «monachiser la condition des nobles, leur imposer les interdits et les obligations des religieux, de vivre chastement, de chanter les Psaumes»" ${ }^{32}$. Quoi qu'il en soit, la précocité de la vocation monastique de Guillaume, son vou de se retirer au cloître de Jumièges, son souci de ne parler de son projet qu'à de rares confidents, l'opposition et les pressions de son entourage, l'obéissance à la volonté du père jusqu'au jour où, Rollon n'étant plus de ce monde, Guillaume décide de franchir

30. Ibid., p. 315-317.

31. «Christianae religionis summa trimodo constat ordine distincta: munifico laicorum canonicorumque atque monachorum labore exercita" (Dudon, p. 201). Nous ne pensons pas que Dudon s'inspire ici de la théorie de tripartition sociale d'Heiric d'Auxerre, comme on en a parfois émis l'hypothèse (Lecouteux, Stéphane, "À partir de la diffusion... ", p. 6 et 10). Si, chez Heiric, l'esprit est le même et si la primauté est également accordée à l'état monastique, sa formulation est différente, dans la mesure où elle procède d'un classement par catégories fonctionnelles et non de type canonique (oratores, belligerantes, agricolantes).

32. Duby, Georges, Les trois ordres ou l'imaginaire du féodalisme, Paris, Gallimard, 1978 (rééd.1996), p. 74 . 
le pas et de choisir sa propre voie, tout cela offre un parallélisme remarquable avec les situations qu'Annon met en scène dans sa biographie de saint Hugues, autour du thème de la vocation monastique contrariée. La seule différence est que Guillaume, lui, ne verra pas se réaliser son vœu de finir ses jours au sein de la communauté de Jumièges; c'est par une voie plus sublime encore, celle du martyre, qu'il gagnera sa place au sein du peuple des élus. Quant au thème du partage du repas si cher à Annon, il n'est pas non plus absent de la Vita Guillelmi. À titre d'exemples, citons la fastueuse réception organisée par Guillaume Longue-Épée pour la conférence de Lyons-la-Forêt, le grand banquet offert par le comte Herluin au château de Montreuil après la victoire ${ }^{33}$, ou encore l'anecdote édifiante de l'invitation des moines de Jumièges maladroitement refusée par Guillaume (deux versions), cette dernière offrant un parallèle évident avec le passage ci-dessus mentionné de la Vie de saint Eucher ${ }^{34}$.

Ces similitudes sont celles qui sont les plus visibles à la première lecture, mais il y en a beaucoup d'autres, que nous ne pouvons toutes énumérer ici. Précisons toutefois qu'elles ne se limitent pas toujours à des correspondances thématiques. À plusieurs reprises, dans les textes présumés correspondre à des œuvres dérivées de la Vita Guillelmi, nous avons reconnu les mêmes termes ou les mêmes expressions rares que ceux utilisés par l'auteur des Vies des saints de Jumièges: il s'agit là, selon toute vraisemblance, de fragments de l'œuvre originale ayant échappé à la refonte ${ }^{35}$. Chez Guillaume de Jumièges, il semble même que certains passages aient été pratiquement recopiés mot à mot sur la Vita Guillelmi. C'est notamment le cas de sa description d'une «entrée» ducale à Rouen, ce passage portant la marque, reconnaissable entre toutes, du style d'Annon: exceptionnellement, il semble que Guillaume de Jumièges ait préféré ici le récit original à sa réécriture par Dudon de Saint-Quentin ${ }^{36}$.

En présence de recoupements aussi nombreux, une conclusion s'impose: l'auteur de la Vita prima Guillelmi n'est probablement autre qu'Annon, abbé de Jumièges et de Saint-Mesmin de Micy (942-973). Sans doute écrit au scriptorium de l'abbaye ligérienne, donc après les environs de 950, ce texte est nécessairement antérieur à 963, date de la mort du comte de Poitiers Guillaume Tête d'Étoupe, lequel est mentionné dans le Planctus comme étant encore vivant

33. Dudon, III, 47 et 64 , p. 192 et 204.

34. Selon Guillaume de Jumièges, l'anecdote se place avant la restauration de l'abbaye, et ce sont les deux moines d'Haspres qui invitent Guillaume à partager leur frugal repas (GND, t. 1, p. 86). Dans le récit de Dudon de Saint-Quentin, l'épisode se situe après la rencontre entre Guillaume Longue-Épée et l'abbé Martin (Dudon, p. 202).

35. En dépit de l'ampleur et du brio de son travail de réécriture, Dudon de Saint-Quentin lui-même laisse parfois échapper une formule trahissant sa dépendance à l'égard du texte d'Annon. Comparer ceci, par exemple: "pro stabilitate sacrosanctae Ecclesie sanctaeque fidei et pacis proque fidelitate sui [regis]...» (Dudon, IV, 70, p. 224) au passage suivant de la Vita Hugonis: "pro animabus fidelium et regia incolomitate simulque pro statu sanctae Dei ecclesiae et trium ordinum stabilitate» $(\mathrm{VH}, 27)$. De même, la formule peu usitée «mundialibus aerumnis» (Dudon, IV, 121, p. 283) se retrouve chez Annon ("aerumnis mundialibus », VH, 12; "aerumnas mundiales », ibid., 20; «erumnis mundialibus », ibid., 29).

36. Voir ci-dessous, annexe II. 
et fut vraisemblablement, avec Richard $\mathrm{I}^{\mathrm{er}}$, un des deux destinataires officiels de la Vita. Euvre particulièrement ambitieuse, pour ne pas dire courageuse car elle va totalement à rebours de l'opinion de l'époque, les Normands de Rouen étant encore considérés en Francia comme à peine plus fréquentables que leurs pères qui naguère avaient mis le royaume à feu et à sang, la Vita Guillelmi a pour but de célébrer non seulement les vertus chrétiennes du refondateur de l'abbaye de Jumièges, de l'apôtre de la non-violence et du protecteur des humbles qu'a été le comte Guillaume, mais, plus encore, celles d'un prince qui a offert sa vie en sacrifice pour la paix dans le royaume. D'une certaine façon, ce descendant de Vikings a même ainsi dépassé le modèle de vertu militante incarné par Géraud d'Aurillac, en qui certains contemporains de son biographe Odon refusaient de voir un saint parce qu'il n'avait pas connu le martyre ${ }^{37}$.

Les données textuelles ne sont pas suffisantes pour nous permettre de savoir si le duc Guillaume avait eu réellement l'intention de se retirer dans un monastère à la fin de ses jours. Le fait est certes tout à fait plausible, une telle pratique étant alors courante dans les milieux princiers, sur le continent comme en Angleterre. En tout cas, il semble bien que le bruit en ait couru à l'abbaye de Jumièges dès 942 et que les moines de cet établissement aient eu bon espoir de voir leur monastère devenir le sanctuaire dynastique des comtes de Rouen, à l'instar de celui de Saint-Cyprien pour les comtes de Poitiers. Annon avait donc là une occasion d'exalter les vertus monastiques et d'offrir à ses deux protecteurs laïques, le duc de Normandie Richard I $^{\text {er }}$ et le comte de Poitiers Guillaume Têted'Étoupe (qui allait finir ses jours sous l'habit monastique à Saint-Cyprien), un «miroir du prince» conforme à l'idéologie politique des premiers clunisiens. Au terme d'une longue période de tension diplomatique entre le royaume de France et la Normandie, résultant de la capture en 945 du roi Louis IV par Hugues le Grand avec la complicité des Normands de Rouen, la fin tragique de Guillaume était aussi pour Annon, une quinzaine d'années environ après l'événement, une occasion de tirer parti de l'émotion publique encore très vive à la suite de cet assassinat. Dans une phrase que son remanieur Dudon, plutôt enclin à gommer tout ce qui, dans la Vita prima, évoquait une sujétion trop étroite au roi de France, a visiblement laissé échapper, il n'hésite pas à affirmer que le duc de Normandie avait payé de sa vie sa fidélité à Louis IV d'Outremer ${ }^{38}$. De même, les Vies de saint Hugues et de saint Eucher comportent l'une et l'autre un hommage très appuyé à la dynastie carolingienne. Il semble donc que les œuvres d'Annon

37. D'où cette mise au point agacée d'Odon: "At vero illi delirant, quod nec martyr, nec confessor valeat dici, sciant quod utrumque dici possit » (Vita Geraldi, préface du livre II, P. L., t. 133, col. 669).

38. «Audiens autem rex Franciae Luthdovicus quod, Arnulfi Flandrensis comitis versutia deceptus, pro stabilitate sacrosanctae Ecclesie sanctaeque fidei et pacis, proque fidelitate sui martyrizatus esset dux Northmannorum Willelmus...» (Dudon, IV, 70, p. 224). Le lien est évident entre cette phrase de Dudon et le passage de l'Histoire de Richer où, de manière tragiquement prémonitoire, Guillaume se déclare prêt à mourir pour le roi Louis IV (cf. ci-dessus, note 14). La formule pro stabilitate sacrosanctae Ecclesie sanctaeque fidei et pacis est probablement une reprise littérale du texte d'Annon (voir ci-dessus, note 35 ). 
s'inscrivent dans le même courant légitimiste et pro-carolingien que la Vita secunda de saint Romain, la Vita tertia de saint Ouen et la Vie de sainte Clotilde, toutes trois composées vers 960 à la demande de l'archevêque de Rouen Hugues (942-989), par un certain Gérard que nous avons récemment proposé d'identifier à un abbé de Saint-Crépin de Soissons ${ }^{39}$.

Les études codicologiques montrent qu'aux vies de saints étaient fréquemment associés des résumés de ces Vitae destinés à un usage liturgique et se présentant sous la forme d'offices, de leçons, d'hymnes ou de sermons. Très souvent, comme c'est le cas pour le Sermo de festivitate sancti Geraldi composé par Odon de Cluny pour accompagner la Vita Geraldi, ces pièces liturgiques étaient réalisées par l'hagiographe lui-même, dans la foulée de l'écriture de la Vita ${ }^{40}$. Passer de la rédaction d'un texte en prose à celle d'un poème rythmique ou d'un chant, réaliser une version abrégée de son propre ouvrage ou écrire soi-même les titres des chapitres, autant d'exercices qui étaient normalement à la portée de chaque auteur. Ceci jette un éclairage nouveau sur l'origine du Planctus de Guillaume Longue-Épée. Selon toute probabilité, ce chant funèbre fut composé pour être joint à la Vita Guillelmi, le tout formant un livret que l'on ressortait en différentes occasions, notamment au jour anniversaire de la mort du duc. Dans ces conditions, vu le grand intérêt qu'Annon manifeste pour les chants et les hymnes tout au long de ses œuvres hagiographiques - au point de terminer la Vita Hugonis par un chant sans doute de sa propre composition (un florilège des Psaumes) -, il est logique de se demander s'il n'est pas lui-même l'auteur du Planctus ${ }^{41}$. On n'arrivera probablement jamais, sur ce point, à une certitude absolue. Il n'en reste pas moins qu'entre la Complainte et les Vies des deux abbés de Jumièges, les similitudes linguistiques sont troublantes. Nous avons relevé une vingtaine de concordances lexicales, ce qui est beaucoup, surtout si l'on tient compte de la brièveté de la Complainte et de son appartenance à un genre littéraire différent de celui dont relevait la Vita en prose. Ajoutons que plusieurs concordances portent sur des mots rares comme archanum, insons, prestol(ari), levius, singultus, conglobati, et que d'autres similitudes apparaissent au niveau des expressions, par exemple dans la manière de calculer le temps en comptant les jours (insequenti die, tertia die, sexta die...), ou d'évoquer la dédicace d'une église (monasterium in honore... dicatum) ${ }^{42}$.

39. Le Maно, Jacques, «La réécriture hagiographique au $\mathrm{X}^{\mathrm{e}}$ siècle; autour des Libri Gerardi de la cathédrale de Rouen", in La place de la Normandie dans la diffusion des savoirs: du livre manuscrit à la bibliothèque virtuelle, Congrès des Sociétés historiques et archéologiques de Normandie, 11, Actes du congrès d'Avranches (octobre 2006), Rouen, Fédération des Sociétés historiques et archéologiques de Normandie, 2006, p. 23-36.

40. Iogna-Prat, Dominique, «La Vita Geraldi d'Odon de Cluny; un texte fondateur?», in Guerriers et moines..., p. 153.

41. C’est également, mais pour des raisons différentes des nôtres - la Complainte aurait été rédigée à Jumièges, peu après la mort du duc -, l'hypothèse envisagée par Elisabeth van Houts: «abbot Anno of Jumièges and Micy (d. 973) may well have been the author (of the planctus)» (GND, t. 1, p. XXIX, n. 43).

42. Voir ci-dessous, annexe I. 


\section{Quelques aspects éditoriaux}

Puisque nous sommes maintenant en mesure d'attribuer au moins quatre œuvres à l'abbé Annon (cinq si l'on ajoute le Planctus), l'étape suivante est de chercher à savoir dans quel ordre elles furent réalisées. Dès lors, en effet, il devrait être possible de distinguer, au sein de la Vita Guillelmi, les éléments qui sont une simple reprise des écrits précédents de ceux qui correspondent à de nouveaux thèmes, réexploités par l'auteur dans ses œuvres suivantes. En l'état actuel des recherches, divers indices suggèrent que notre auteur pourrait avoir commencé par la Vie de saint Aycadre. Ce choix s'expliquerait aisément, les moines de Poitiers installés à Jumièges ayant une dévotion particulière pour cet ancien abbé en raison de ses origines poitevines; à leur arrivée en Normandie, ils en firent le second patron de l'abbaye après saint Pierre ${ }^{43}$. La Vita Guillelmi et le Planctus viendraient en deuxième et troisième positions, suivis de la Vita Hugonis et de la seule biographie orléanaise du corpus, la Vie de saint Eucher.

Ce premier essai de classement chronologique des œuvres apporte un éclairage intéressant sur la méthode de travail d'Annon. L'auteur avait beaucoup de mal à donner une consistance historique à la figure de saint Hugues, pour lequel il ne disposait d'aucun autre élément que deux brèves mentions, d'ailleurs contradictoires, dans des annales carolingiennes ${ }^{44}$. Pour construire son récit, il n'hésita donc pas à en calquer une grande partie sur sa biographie précédente, dotée en principe d'une trame historique plus solide, la Vita Guillelmi: n'étaitce pas, là aussi, l'histoire d'un homme partagé entre son désir de se faire moine et le devoir de rester à la place qui, par sa haute naissance, lui avait été assignée dans la société? De là provient sans doute le remarquable parallélisme, déjà noté, entre les scènes de l'enfance de saint Hugues et celles qui ont trait, dans la Vita Guillelmi, aux jeunes années de Guillaume. De là aussi le fait que se retrouvent dans la Vita Hugonis plusieurs références aux trois ordres de la société. Cette notion était déjà présente dans la biographie de Guillaume, où la théorie de la tripartition sociale était, comme nous l'avons vu, expliquée par la bouche de l'abbé Martin; mais, et ceci montre bien, nous semble-t-il, que la Vita Hugonis fut écrite postérieurement, elle n'est évoquée dans cette dernière œuvre que de manière allusive, comme si Annon, ayant exposé sa théorie dans la Vita Guillemi et considérant désormais la notion comme acquise, n'avait pas jugé utile de refaire la démonstration. Un troisième cas de répétition thématique, particulièrement intéressant dans la mesure où il touche à la question très

43. Le Maнo, Jacques, «Autour de la renaissance monastique... », p. 298.

44. Il s'agit des Annales Mettenses priores, où figure la mention d'Hugues, petit-fils de Pépin II et fils de Drogon et d'Anstrude (éd. Bernhard von Simson, MGH, Scriptores rerum germanicarum in usum scholarum, 10, Hanovre et Leipzig, Hahn, 1905, p. 16), et de la Vita Karoli d'Éginhard, citant un autre Hugues comme fils de Régine, concubine de Charlemagne (éd. Georg Heinrich Pertz et Georg Waitz, MGH, Scriptores rerum germanicarum in usum scholarum, 25, Hanovre et Leipzig, Hahn, 1911, p. 23). Annon chercha à concilier ces deux données, ce qui n'était évidemment pas une bonne solution: s'il tomba juste en donnant à la mère de son personnage le nom d'Anstrude - car le vrai saint Hugues fut bien celui des Annales Mettenses -, en revanche Annon fit le mauvais choix en l'identifiant au fils de Charlemagne. 
controversée des origines de la mère de Guillaume Longue-Épée, concerne la mère de saint Hugues. Lorsqu'Annon nous dit qu'elle était une fille de Tassilon de Bavière, qu'elle avait été emmenée à la cour de Charlemagne après la défaite de Tassilon et son exil à Jumièges, que l'empereur l'avait prise pour femme et qu'elle avait élevé toute seule son fils jusqu'à l'âge de quatre ans, il s'agit là d'une belle série d'affabulations, apparemment sortie toute entière de l'imagination de l'auteur. Une fois rapproché de la Vita Guillelmi, ce récit prend néanmoins une autre signification: on constate alors qu'il ressemble étrangement à l'histoire de Poppa, fille d'un comte ou d'un marquis neustrien, enlevée par le chef viking Rollon au cours d'un de ses raids en Francia; de Rollon, elle eut un fils qu'elle baptisa sous le nom de Guillaume et éleva dans la religion chrétienne, jusqu'à ce que Rollon, dans l'intention d'en faire son successeur, décidât de prendre en charge son éducation.

Si aucun exemplaire de la Vita prima Guillelmi n'est parvenu jusqu'à nous, il semble cependant possible de déceler les traces de plusieurs manuscrits perdus. Le texte-souche, que nous proposons d'appeler le «ms $\alpha$ », fut vraisemblablement réalisé à Saint-Mesmin de Micy. C'est sur une pièce jointe à ce texte que devait se trouver la Complainte qui est à l'origine de la tradition orléanaise sur le Planctus de Guillaume, représentée par le manuscrit du fonds Libri de la Bibliothèque laurentienne de Florence ${ }^{45}$. Nous pensons également avoir retrouvé une trace de la Vita Guillelmi de Micy à travers la présence à Bamberg (Allemagne) d'un livret hagiographique du $\mathrm{X}^{\mathrm{e}}$ siècle contenant les Vies des saints Philibert, Aycadre et Hugues de Jumièges ${ }^{46}$. En effet, c'est dans ce même fonds de Bamberg, dont le noyau fut constitué par des ouvrages provenant de la bibliothèque personnelle de Gerbert d'Aurillac, qu'est conservé le manuscrit autographe de l'Histoire de France de Richer de Reims, élève de Gerbert. Or, nous savons qu'à l'époque où, à la demande de son maître, Richer entreprit la rédaction de cet ouvrage (entre 991 et 996), Gerbert avait pour ami et correspondant un certain Constantin, alors abbé de Saint-Mesmin-de-Micy (v. 990-av. 994), et qui lui envoyait régulièrement des livres ${ }^{47}$. Il est peu probable que le grand avocat de la cause cléricale qu'était Gerbert, écolâtre de la cathédrale de Reims, puis archevêque (991-997), mais aussi auteur à cette époque d'une violente diatribe contre le pape, ait été particulièrement intéressé par les œuvres hagiographiques

45. Bibliotheca Mediceo-Laurenziana de Florence, Ms Libri 30, folios 21v-22v.

46. Bamberg SB ms Patr. 134 (précédemment B.V. 25). Sur ce manuscrit, voir John HowE, "The Hagiography of Jumièges (Province of Haute-Normandie)", (Sources hagiographiques de la Gaule VI), in L'hagiographie du haut moyen âge en Gaule du Nord, manuscrits, textes et centres de production, Martin Heinzelmann (dir.), Beihefte der Francia, Band 52, 2001, p. 121.

47. Sur Constantin, sur ses relations épistolaires avec Gerbert et ses envois de livres à Reims, voir Karl-Ferdinand Werner, art. "Constantin von Fleury", in Lexikon des Mittelalters III, col. 169, et Pierre Riché, Gerbert d'Aurillac, le pape de l'An mil, Paris, Fayard, 1987, p. 78, 93 et 208. Cependant, les dates données par ces deux auteurs pour l'abbatiat de Constantin à Micy sont fautives et doivent être corrigées en fonction des observations de Robert-Henri BAUTIER et Gillette LABORY, "Introduction», Helgaud de Fleury, Vie de Robert le Pieux, Paris, Éditions du CNRS, 1965, p. 23, n. 4, à compléter par les remarques de Thomas HeAD, Hagiography and the Cult of Saints - The Diocese of Orléans, 800-1200, Cambridge, Cambridge University Press, 1990, p. 240. 
d'Annon, les thèses défendues dans celles-ci étant toutes à l'opposé exact des siennes ${ }^{48}$. En revanche, la Vita Guillelmi du même Annon était susceptible de rendre de grands services à son élève Richer. Pour les chapitres de son ouvrage couvrant la période des années 930 à 942, elle apportait, sur le deuxième duc de Normandie, nombre d'informations inconnues de Flodoard, sa source principale. Il est donc fort possible que les emprunts de l'Histoire de Richer à la Vita Guillelmi proviennent d'un manuscrit inséré dans le même livret que les trois Vies de saints de Jumièges, livret qui aurait été envoyé par l'abbé de Micy à son ami Gerbert vers le début des années 990. La Vita Guillelmi en aurait été détachée par la suite, à une époque où il n'était plus jugé convenable de conserver la biographie d'un prince laïque, fût-il un «martyr», parmi les vies des saints; c'est pour la remplacer qu'aurait alors été réunie aux trois vies de Jumièges une pièce de format légèrement différent et d'une écriture plus tardive, le Liber scintillarum de Defensor de Ligugé.

L'abbaye de Jumièges, fondation qui avait été chère au cœur du duc disparu, et à laquelle était destinée une partie importante de la production du scriptorium de Micy dans la seconde moitié du Xe siècle, fut certainement très tôt en possession d'un exemplaire de la Vita Guillelmi. Ce texte, que nous proposons d'appeler le «ms $\beta$ », est celui qui fut utilisé par Guillaume de Jumièges pour ses compléments et ses correctifs au récit de Dudon de Saint-Quentin. On en retrouve aussi quelques éléments dans les Annales de Jumièges ( $\mathrm{XI}^{\mathrm{e}}$-XIII ${ }^{e} \mathrm{~s}$.), sans que l'on puisse déterminer s'ils procèdent en l'occurrence de la Vita Guillelmi elle-même ou de traditions dérivées. Un troisième exemplaire («ms $\gamma »)$ a dû exister à la cathédrale de Rouen, lieu d'inhumation de Guillaume Longue-Épée. C'est probablement ce manuscrit qui fut entre les mains de Dudon de Saint-Quentin lorsque, au cours de son séjour à Rouen auprès de Richard $\mathrm{I}^{\text {er }}(\dagger 996)$ et de l'archevêque Robert (989-1037), il commença à réunir la documentation pour sa geste des deux premiers ducs. Autre indice de la présence d'une copie de la Vita Guillelmi à Notre-Dame de Rouen, l'œuvre semble également avoir été connue du premier rédacteur des Annales de Rouen, rédigées à la cathédrale avant $1087^{49}$. Sachant que le comte de Poitiers Guillaume ( $†$ 963) fut probablement, avec Richard I ${ }^{\text {er }}$ de Normandie, un des deux destinataires de la Vita, il serait également assez logique qu'un quatrième manuscrit ait pris la direction du Poitou. Cependant, nous n'avons pas encore trouvé d'élément probant en ce sens; il n'est certes pas exclu que le manuscrit 240 de Clermont-Ferrand, seul témoin connu du Planctus de Guillaume en dehors du texte de Florence, soit issu d'une filière poitevine, mais cela reste pour l'instant une simple hypothèse de travail.

Avec un minimum de trois ou quatre manuscrits, des lieux de conservation qui se répartissent entre l'Orléanais, la région rouennaise et Reims, sans compter le possible envoi de copies en d'autres lieux par l'intermédiaire des prieurés de Micy et de Jumièges, on constate que la diffusion de la Vita prima

48. En particulier dans la Vita Hugonis, où le personnage du pape est magnifié et où il est clairement dit qu'il vaut mieux être simple moine à Jumièges qu'archevêque à Rouen.

49. Cf. ci-dessus, note 11. 
Guillelmi - étroitement comparable, comme on pouvait s'y attendre, à celle des Vies de saint Aycadre et de saint Hugues - fut loin d'être négligeable. Ceci rend d'autant plus étonnant, de prime abord, l'oubli dans lequel cette œuvre est tombée. La chose est toutefois moins surprenante quand on sait quel succès rencontra, dès le $\mathrm{XI}^{\mathrm{e}}$ siècle, l'ouvrage du principal remanieur de la Vita, Dudon de Saint-Quentin.

Dans un célèbre passage du prologue de ses Gesta, Dudon rapporte qu'un beau jour, le duc Richard I ${ }^{\text {er }}(\dagger 996)$ lui demanda d'écrire l'histoire de son grandpère Rollon et de l'établissement des Normands en Neustrie ${ }^{50}$. Bien que la Vita Guillelmi d'Annon ait sans doute comporté un certain nombre d'informations sur le père et la mère de Guillaume, éventuellement aussi sur le baptême de Rollon et sur le traité de Saint-Clair-sur-Epte ${ }^{51}$, on considérait peut-être, à la cour de Rouen, qu'il manquait encore une vraie biographie du fondateur de la dynastie. Il est également possible que la commande de Richard $\mathrm{I}^{\mathrm{er}}$ ait répondu au souhait de renouveler la Vita Guillelmi en réalisant une version moins dépendante du modèle hagiographique des passiones et plus proche de l'écriture historiographique. Le silence de Dudon à ce sujet n'aurait rien d'étonnant: il ne serait pas le premier auteur à faire passer pour une oeuvre originale ce qui n'est en réalité, pour l'essentiel, qu'un travail de réécriture. Lorsqu'il entreprit la rédaction définitive de ses Gesta - pas avant 1013/1014 pour certains chapitres, si l'on retient une hypothèse d'Éric Christiansen $-{ }^{52}$, la Vita Guillelmi fut, en tout cas, une source très précieuse pour Dudon. De ce texte furent tirés la plus grande partie de son livre III, plusieurs passages de son livre II consacré à l'histoire de Rollon et le début du livre IV sur l'enfance de Richard ${ }^{\mathrm{er}}$. Ces reprises impliquaient, en revanche, d'importants remaniements.

Chez Annon, la syntaxe laisse en effet beaucoup à désirer ${ }^{53}$, et il est certain qu'une œuvre telle que la Vita Guillelmi était susceptible d'attirer de sévères critiques de la part du cénacle de lettrés qui, vers l'an Mille, s'était constitué autour de l'archevêque de Rouen Robert (989-1037): on y cultivait l'art du beau discours et de la formule bien ciselée, mais on y avait la plume acérée et le jugement était sans complaisance à l'égard des œuvres de certains collègues ${ }^{54}$. C'est précisément pour des raisons stylistiques, semble-t-il, que les moines de Jumièges décidèrent, vers le milieu du $\mathrm{XI}^{\mathrm{e}}$ siècle, de faire réécrire la Vie de saint Aycadre d'Annon. Ils confièrent ce travail à un certain Fulbert, probablement un maître de l'école du chapitre cathédral de Rouen, qui, dit-il, trouva la Vita existante tellement

50. «scilicet ut mores actusque telluris Normannicae, qui etiam et proavi sui Rollonis, quae posuit in regno jura describerem " (Dudon, prologue, p. 119).

51. Ces deux derniers points, d'une importance historiographique majeure s'il s'avère que, plusieurs décennies avant Dudon de Saint-Quentin, ils étaient déjà traités dans la Vita Guillelmi d'Annon, sont actuellement en cours d'étude.

52. Dudon, trad. Christiansen, op. cit., p. 221, n. 424.

53. Van der Straeten, Joseph, "Vie inédite de saint Hugues... », p. 231.

54. Musset, Lucien, «Le satiriste Garnier de Rouen et son milieu (début du XI siècle)», Revue du Moyen-Âge latin, X, 1954, p. 237-266 (paru 1956). 
mauvaise que la seule idée de devoir la réécrire lui donna des cauchemars ${ }^{55}$. Si l'on considère que l'objectif de Dudon était de réaliser un ouvrage historique à l'intention des membres de la cour, ceci ne pouvait se faire, de toute façon, sans une importante transformation formelle du texte d'Annon. À en juger par le mode d'écriture de ce dernier dans les Vies de saint Aycadre et de saint Hugues - les deux œuvres qui, en l'état actuel des recherches, semblent encadrer chronologiquement la biographie de Guillaume -, celle-ci devait être toute entière émaillée de citations des Psaumes et de commentaires spirituels. Or, si un tel discours était conforme aux canons de l'écriture hagiographique, il était, en revanche, mal adapté aux exigences de l'historiographie. On peut donc penser que Dudon de Saint-Quentin effectua à cet égard un sérieux toilettage de l'œuvre d'Annon, en ne conservant que quelques topoi hagiographiques utiles à sa propre narration. Les deux «visions» de Rollon, père de Guillaume LongueÉpée, en font sans doute partie, mais il est significatif que ces épisodes miraculeux soient justement parmi les rares passages que Guillaume de Jumièges n'a pas souhaité reprendre dans ses Gesta, estimant qu'ils ne contribuaient guère à la crédibilité du récit ${ }^{56}$.

D'autres raisons, plus profondes car elles touchent à la thématique et au projet même de la Vita Guillelmi, peuvent expliquer l'importance des remaniements opérés par Dudon. Comme nous l'avons vu, l'œuvre devait être principalement axée sur une valorisation de l'idéal monastique, les scènes-clés étant celles où l'on voit Guillaume s'interroger sur son salut et préparer son retrait de la vie publique pour entrer au cloître. Or, si un tel discours avait tout pour être bien reçu dans les milieux proches de la mouvance des Clunisiens, il devait passer plus difficilement auprès des lettrés de la petite «académie» nouvellement constituée à Rouen dans l'entourage de l'archevêque Robert. Ces intellectuels dont faisait partie Dudon de Saint-Quentin, grand admirateur, rappelons-le, d'Adalbéron, évêque de Laon connu pour ses violentes diatribes contre l'ordre monastique, étaient les représentants d'une culture cléricale qui n'avait guère d'affinités avec les idéaux mis en avant par l'abbé Annon dans ses écrits. Lorsque Dudon modifie l'énoncé du schéma trifonctionnel d'Annon, de telle sorte que l'ordre des moines se trouve rétrogradé de la première à la troisième position, cette petite manipulation n'a certainement rien d'anodin ${ }^{57}$. Sur d'autres points, il semble que la réécriture de Dudon ait eu pour but de recadrer politiquement les récits d'Annon, notamment lorsqu'ils touchaient à des questions d'État hautement sensibles, comme celle des relations des comtes de Rouen avec le Roi. Que, de ce dernier point de vue, le contexte ait évolué depuis la rédaction de la Vita prima, cela transparaît parfois à travers une simple anecdote: aux gestes d'extrême déférence des personnages d'Annon en présence du souverain ( $Q u i$ veniens, ut mos habetur regius, ante pedes eius sae prostravit », VH, 8 ; "Ipsi vero cum velocitate eius praesentia prolati, pedes ipsius advoluti», VE, 4), s'oppose

55. Le Maно, Jacques, "Autour de la renaissance monastique... », p. 321-322.

56. GND, t. 1, p. 7 .

57. Cf. ci-dessus, note 31 . 
chez Dudon la fameuse scène de la rencontre de Saint-Clair-sur-Epte, où, dans une situation analogue, le roi Charles est tourné en ridicule ${ }^{58}$. En cette période des environs de l'an Mille, marquée dans le duché par le développement d'une culture identitaire et le début de l'affirmation d'un sentiment national normand ${ }^{59}$, la conception carolingienne du monde telle qu'elle avait été défendue, non sans un certain angélisme, par l'abbé Annon, c'est-à-dire celle d'un peuple chrétien unique et sans frontières, rassemblé dans un même amour de la paix et de la justice autour du Roi, devait sembler quelque peu dépassée à la cour de Rouen.

Quoiqu'il en soit, le large succès éditorial que connut l'ouvrage de Dudon, non seulement en Normandie, mais aussi dans une large partie de la moitié nord de la France, fut probablement fatal à la carrière de la Vita prima Guillelmi. Dès la seconde moitié du XI ${ }^{\mathrm{e}}$ siècle, il est clair que le chroniqueur Guillaume de Jumièges préférait la version de Dudon à la version primitive, cette dernière n'étant plus bonne, en l'occurrence, qu'à apporter quelques compléments aux récits du chanoine. Comme souvent, la réécriture avait donc chassé l'original.

\section{Annexe I - Similitudes linguistiques entre la Complainte sur la mort} de Guillaume et les Vies des saints Aycadre et Hugues:

a) Correspondances lexicales:

- «... matre quoque consignata alma fide...» (Complainte, II); «... suaque benedictione consignatis omnibus... $(\mathrm{VH}, 24)^{60}$.

- «... regum more...» (Complainte, IV); « ... more regio...» $(V H, 7)$.

- «... sibi fecit seniorem regnaturum...» (Complainte, IV); «... in contubernio sanctorum manet regnaturus cum Domino» $(V H, 21)$; « ... per secula sempiterna regnaturi cum eo» $(V A, 63)$.

- «... monasterium... quod dicatum sancti Petri in honore...» (Complainte, $\mathrm{VI}) ;$ «... oratorium... in honore almae Dei Genitricis Mariae gloriosissimae... dicatum» $(V A, 9)^{61}$.

- «... tantum nefas intimare cunctis damnum...» (Complainte, VII); «...pro tanto geminali dampno gemebat...» $(V A, 68)$.

58. $V H, 8$.

59. BAuduin, Pierre, «Autour d'une construction identitaire: la naissance d'une historiographie normande à la charnière des $\mathrm{X}^{\mathrm{e}}-\mathrm{XI}^{\mathrm{e}}$ siècles", Cahiers du GRHIS (Groupe de Recherche d'Histoire de l'Université de Rouen), ${ }^{\circ}{ }_{13}, 2001$ (Actes du Colloque: Conquête, acculturation, identité: des Normands aux Hongrois. Les traces de la conquête), p. 79-89.

60. Le vers de la Complainte est difficile à traduire littéralement. Dans le mot consignata (du verbe consignare), il doit y avoir l'idée que la mère de Guillaume était marquée du signe de la religion chrétienne.

61. Dicatum, du verbe dicare, dédier («...et fundamenta illius domus sua sancta benedictione dicaret »,VA, 9). Dans la Complainte, la leçon dicatur est également recevable, mais certains auteurs semblent avoir cru, à tort, à une graphie fautive du subjonctif dicetur. 
- «cum singultu et ploratu recitandum»(Complainte, VII); «pro cantu gemitum singultuose ederent» $(\mathrm{VH}, 29)$.

- «Impetrata ad loquendum sexta die,...» (Complainte, IX); «Tumque licentia a magistro impetrata,...» $(V A, 2)^{62}$.

- «... ad insontem properavit...» (Complainte, IX); «... et parce hic insonti gregi...» $(V A, 59)^{63}$.

- «... ad insontem properavit... (Complainte, IX); «... ut quantocius properaret ad eum...» $(V H, 15)$; «Coepit properare ad domum» $(V H, 15)$; «ad hoc... ilico properavit»; «ad obedentiam properavit» $(V A, 43) ;$ «... ad finem properare dispositum...» $(V A, 63)$.

- «... levius [ut] mactaretur» (Complainte, IX); « ... ut abscessum Patris eorum levius ferrent» $(V A, 70)^{64}$.

- «Insequenti namque die...» (Complainte, $\mathrm{X})$; «Eadem nanque die...» (VA, 38). «Quadam nanque nocte...» $(V A, 55)$.

- «Insequenti namque die conglobati...» (Complainte, $\mathrm{X}) ;$ «... pariter in unum conglobati...» $(V A, 60)$; «... eos undique... conglobatim adunari...» $(\mathrm{VH}, 17)^{65}$.

- «... archanum/sibi ipsi atque vobis profuturum...» (Complainte, XII); «... secretorum archanis...» $(\mathrm{VH} \text {, prologue })^{66}$.

- «Hoc cis intuens...» (Complainte, XII); «Qui eum intuens,...» $(V H, 4)$; "Intuentes autem illum...» $(V H, 15)$; «Quos ut sanctus Dei intuens...» $(\mathrm{VH}, 26)$.

- «... expectante prestolando» (Complainte, XII) : «... prestulaturus Domini adventum» $(\mathrm{VH}, 3 \mathrm{O})^{67}$.

- «... quo jubente, festinavit...»(Complainte, XII); «Iubente autem eum eo, custos pueri adduxit eum...» $(\mathrm{VH}, 4)$; «Iubente autem domno papa, allatus est...» $(V H, 12)$; «iubente eo,... resedit» $(V H, 23)$.

- «...quo jubente, festinavit...»(Complainte, XII); «Angelus Domini bonus festinavit...» $(V A, 63)$.

62. Du verbe impetrare, obtenir; dans l'extrait de la Complainte, il s'agit du rendez-vous qu'Arnoul a obtenu de Guillaume.

63. Insons, peu usité, équivalent d'innocens. La formule de prière parce hic insonti gregi de la Vie de saint Aycadre est probablement inspirée d'un passage de l'Exode: «...insontem et justum non occides..." (Exode 23, 7).

64. De leve, terme peu usité, employé par Annon à la place de l'adverbe leviter («...onusque leve suscipiens...", VA, 4; "...onus ejus leve suscepit... », ibid., 47).

65. Conglobatus, du verbe conglobare, rassembler. Sur le manuscrit de Florence, il est écrit conglobamti. C'est certainement par erreur que Philippe Lauer restitue conglobanti.

66. Arcanum, secret; noter, dans les deux cas, la même orthographe inhabituelle archanum.

67. Du verbe praestolari, attendre (peu usité). 
- «... corpus terre, flatum celo/... obtulerunt» (Complainte, XIV); "uti os et caro... flatu sancto connexi» $(V A, 14)$; «flatus Dei consolatorius» $(V H, 17)$; «sanctuum vestrorum flatuum» $(V H, 23)$.

- «Erant orbis obtimates...» (Complainte, XV); «rex... cum suis optimatibus» $(\mathrm{VH}, 17)$; «cum rege suisque optimatibus» (ibid., 27); «... omniumque regis optimatum...» (ibid., 29).

- «...pauperumque consolator...» (Complainte, XVI); " egregius consolator» $(V H, 23)$; «consolatorem Dominum» $(V A, 67)$.

b) Similitudes d'expressions:

- Redondances: "regnaretque regum more» (Complainte, IV); "expectante prestolando» (ibid., XII); «festinavit propere» $(V A, 57)$; «... pro tanto geminali dampno gemebat...» $(V A, 68)$; «auxiliante et adjuvante eo » $(\mathrm{VH}$, prologue); «viros gloriosos gloriosae statuerunt» $(\mathrm{VH}, 18)$.

- Olim, dans le sens de "précédemment”, “auparavant”: "Hic audac $(t)$ er olim regem Hcludowicum/sibi fecit seniorem» (Complainte, IV); "Decedente... Karolo,... omni mundo olim eius ditioni subacto luctus...» $(V H, 29)$.

- Tot, à propos d'une abondance de bienfaits, de vertus: «... numerando tot bonorum...» (Complainte, VII); "pro tot spiritualibus donis...» (VA, prologue); «... de tot virtutibus supra memoratis...» $(V H, 28)$.

- Comptage des jours: «sexta die» (Complainte, IX); «insequenti... die» (ibid., $\mathrm{X})$; "die una et altera seu tertia» $(V A, 18)$; "die tertia» (ibid., 23, 38, 97 et 43); «die altero [sic] » (ibid., 30); «die septima» (ibid., 39); «die tertia» (VH, 24); «quarto... die» [sic] (ibid., 30).

- La parole et le cour: "Tantum ore, et non corde...» (Complainte, X); «... in ore ac in ara cordis...» $(V A, 15)^{68}$.

- Succession de qualificatifs en - tor: «Pauperum consolator et... viduarum suffragator» (Complainte, XVI); "praeceptor Dei remuneratorum [sic] et fidelium... orator» $(V A, 15)$; «Memorator malorum et insultator hominum » (ibid., 44).

68. Les termes os et cor marquent la distinction entre ce que l'on dit et ce que l'on pense. Dans la Complainte, il s'agit de montrer le décalage entre les paroles faussement amicales d'Arnoul et son funeste projet; chez saint Aycadre, en revanche, le discours est en parfait accord avec le fond de sa pensée. 


\section{Annexe II - Un fragment présumé de la Vita Guillelmi d'Annon dans les Gesta de Guillaume de Jumièges:}

a) Une entrée du duc Guillaume à Rouen, selon Dudon de Saint-Quentin:

"Quum autem fama celer habitantium in finibus Northmannicis mentes subito de optato Willelmi ducis reditu percelleret, ducemque suum tam magnificum adesse praemoneret, commota nimio gaudio tota Rotomagensium civitas subito processu contra illum exsiliit, diversaque diverticula, ut eum videre posset, requirit, femineoque sexu stante muri in propugnaculis, senilis aetatis vulgo in biviis, juvenilis mediaeque aetatis plebe currente obviam ei: Clerus preastolatus illum in portam civitatis cum reverentia monasticae institutionis ovanter suscepit. " (DuDON, III, 58 , p. 200).

b) Le même épisode d'après Guillaume de Jumièges:

"Quibus regaliter patratis Normannicos limites appetit festinato regressu cum suis. Cuius adventum cunctus prenoscens clerus Rotomagensis ad portas urbis cum laudibus processit obviam illi, civibus utriusque sexus acclamantibus in propugnaculis: "Benedictus qui venit in nomine Domini". Sic inter consonas clericorum seu populorum voces intra Dei genitricis Marie ecclesiam a cunctis inducitur, fusisque Deo precibus ad domum propriam epulaturus properat, stipatus multo comitatu.» (GND, III, 6, éd. van Houts, t. 1, p. 84) ${ }^{69}$.

c) Correspondances lexicales entre la version de Guillaume de Jumièges et les cuvres hagiographiques d'Annon:

- "Quibus regaliter patratis, Normannicos limites appetit festinato regressu...» (GND, III, 6); «Tumque patratis omnibus... quae in itinere erant necessaria,... regressus est ad propria» $(\mathrm{VH}, 13)$.

- «Normannicos limites appetit festinato regressu...» (GND, III, 6); «Sanctus Dei ad propria repedebat con festinatione» $(\mathrm{VH}, 15)$.

- «Cuius adventum cunctus prenoscens clerus,...» (GND, III, 6); «Sentiens autem per spiritum beatus Filibertus illorum adventum, ...» (VA, 22); «Sentiens autem magnus rex adventum filii sui,...» $(V H, 15)$.

69. On voit que la version de Guillaume de Jumièges se démarque nettement du récit de Dudon. Exceptionnellement, il ne s'agit pas non plus d'un résumé du texte du chanoine: les deux versions sont de longueurs à peu près équivalentes. 
- «processit obviam illi» (GND, III, 6); «obviam illis occurrit» $(V A, 35)$; «obviam ei venit quidam» $(V H, 14)$; «misit ei obviam... socios» (ibid., 15); «occurrit ei obviam» (ibid., 16); «ei obviam... venerunt».

- "... civibus... acclamantibus:"Benedictus qui venit in nomine Domini" " (GND, III, 6); "totus grex et reliqua familia... "Benedicti qui veniunt in nomine Domini" decantantes» $(V A, 22) ;$ «... abbas... et tota... familia... dicentes: "Benedicti, qui veniunt in nomine Domini"” $(V A, 30)^{70}$.

- «clericorum seu populorum» (GND, III, 6); «clerum ac populum» $(V H$, 20).

- «intra Dei genitricis Marie ecclesiam» (GND, III, 6); "oratorium... in honore almae Dei genitricis Mariae... dicatum» $(V A, 9)$; «redeunte de basilica beate Dei Genetricis» $(\mathrm{VH}, 26)$.

- «fusisque Deo precibus..." (GND, III, 6); «orationes assiduas fundentes Domino» $(V A, 71)$; «orationes in Domino fudi...» $(V H, 10)$.

- «ad domum propriam epulaturus properat» (GND, III, 6); «Coepit properare ad domum» $(\mathrm{VH}, 15)^{71}$.

- «ad domum propriam epulaturus properat» (GND, III, 6); «mensam adiit epulaturus $(V H, 18)$; «venit ad prandium... epulaturus in timore sancto » (ibid., 21).

- «stipatus multo comitatu» (GND, III, 6); «stipatus eorum sancto collegio» $(V A, 35)$; «stipatus... cum caterva hominum » $(V H, 10)$; «caterva suorum propinquorum stipatus» (ibid., 14).

- «stipatus multo comitatu» (GND, III, 6); «electorum Dei comitatui » (VA, $35)$; "ab his qui in comitatu eius erant" $(\mathrm{VH}, 13)$; "habens in suo sancto comitatu... electos viros» (ibid., 29).

70. L'attribution à l'abbé Annon de la formule "Benedictus qui venit in nomine Domini" de Guillaume de Jumièges apporte peut-être une réponse aux questions soulevées en 1992 par l'éditrice, Elisabeth van Houts: «The significance of William's additions here... is difficult to establish. William obviously describe liturgical acclamations to celebrate victorious homecomings of the Norman dukes, but texts have not survive independently, nor does the sentence "Benedictus... Domini" occur in the earliest surviving Norman laudes regiae» (GND, t. 1, p. 84-85). Le rapprochement avec les extraits des Vies de saint Hugues et de saint Aycadre semble montrer que cette formule de bienvenue était d'un usage courant dans les monastères de la France de l'Ouest au $\mathrm{X}^{\mathrm{e}}$ siècle et qu'il n'était pas propre au rituel des entrées princières.

71. Pour d'autres exemples de la formule properare ad dans les Vies de saint Aycadre et de saint Hugues, voir ci-dessus, annexe Ia. 


\section{Annexe III - Le récit de l'assassinat de Guillaume Longue-Épée dans la Vita depertita, essai de reconstitution du synopsis à partir des textes dérivés :}

- Le destin s'apprête à frapper cruellement le meilleur des hommes:

«Numerando tot bonorum comoramur/tantum nefas intimare cunctis damnum: cum singultu et ploratu recitandum / ovem lupo laniatam... » (Complainte, VII $)^{72}$.

«Refulgebat insuper titulis omnium bonorum, resque adornabat strenue ecclesiarum. Quum autem eniteret augmentatis studiis universae bonitatis... Arnulfus... coepit meditari... mortem ejusdem Willelmi.»(Dudon, III, 61, p. 205) ${ }^{73}$.

- L'instigateur du meurtre est Arnoul de Flandre:

«Erat quidam dives valde plenus fraudis/dicebaturque Arnulfus Flandonensis... » (Complainte, VIII).

«... Arnulfus, dux Flandrensium..., gentisque Franciscae quorumdam principum subdolo consilio et malignitate atrociter exhortatus, coepit meditari et tractare lugubrem mortem ejusdem Willelmi.» (Dudon, III, 61, p. 205).

- Des émissaires sont envoyés à Guillaume pour lui proposer une rencontre:

«Cuius interfectionis series, mox apud conjuratos, ita disponitur, ut ab Ar(nulfo) legati mitterentur, qui pro colloquio multa necessitate in proximo habendo apud Uuilelmum idonee legatione fungerentur. De tempore quererent, quando sibi obveniendum foret» (RICHER, II, 32, p. 121). «Legati itaque directi colloquium petunt et optinent. Tempus post dies XXX datur... Negotioque peracto, legati redeunt.» (RICHeR, II, 33, p. 122)

«... misit legatos expeditionis fraudulentissimae duci Willelmo, qui dicerent, in dolo fraudis nefandissimae, fidelis famulatus charaeque amicitiae si recipere vellet munus.» (Dudon, III, 61, p. 205-206).

72. Ces vers de la Complainte, qui font suite à l'évocation de l'œuvre politique de Guillaume et à l'histoire de la restauration de Jumièges, correspondent sans doute à l'introduction de la troisième partie de la Vita, consacrée au récit de l'assassinat du duc. Dans la littérature hagiographique, il est habituel, avant de raconter les derniers instants du saint, de rappeler combien celui-ci a été bon et vertueux. Plus ses qualités ont été éminentes, plus grande est la perte. S'il est mort assassiné, son âme a été d'autant plus belle que celle de ses meurtriers est noire (v. $₫$ suivant): on retrouve là le thème topique de l'opposition du Bien et du Mal.

73. Les mots «res adornabat strenue ecclesiarum » renvoient à l'épisode de la restauration de l'abbaye de Jumièges, précédemment évoqué par Dudon (1. III, 58). 
- Le lieu choisi pour l'entrevue est une île de la Somme:

«... quodam in flumine...» (Complainte, IX).

"Locum vero secus fluvium Summam peterent " (RICHER, II, 32, p. 121). «Locus quoque in pago Ambianensi secus fluvium Summam ubi est insula Pinchinea conceditur.» (RICHER, II, 33, p. 122).

"Arnulfus vero,... deprecans contra se venire illum, usque ad Pinchiniacum, ut fluenta Somenae essent obstacula inter utrumque exercitum " (DUDON, III, 62-63, p. 207). «Est namque ibi insula, puteulano exaestuantis Somenae gurgite hinc inde et altrinsecus circumdata...» (Dudon, III, 62, p. 207).

- Guillaume accepte l'invitation et propose une trève:

«... Arnulfus Flandonensis/cui se jurejurando sociavit/infelici felix iste... » (Complainte, VIII).

"Consultu ergo Willelmus dux suorum fidelium, dedit Arnulfo comiti sequestram pacem trium mensium, seque ad denominatum mandavit venire placitum » (Dudon, III, 61, p. 206).

- Rendez-vous est pris dans six jours; il n'y aura pas d'échange d'otages:

«Impetrata ad loquendum sexta die/obsideque nullo...»(Complainte, IX) ${ }^{74}$.

«... Tempore ergo constituto...» (RICHER, II, 33, p. 122).

«Statuto imminentis placiti tempore...»(Dudon, III, 61, p. 205-206) ${ }^{75}$.

- Les deux partis se rendent au lieu convenu:

«... ad insontem properavit atrox ille...» (Complainte, IX).

74. Selon Elisabeth van Houts, sexta die signifierait un samedi (GND, t. 1, p. 95, n. 2). Toutefois, dans la manière ecclésiastique et médiévale de compter, le sixième jour correspond au vendredi; pour le samedi qui est un jour spécial, les auteurs, dont Annon lui-même ( $V A, 44 ; V H, 20)$, utilisent habituellement le terme Sabbatum (nous remercions Pascal Pradié pour ces informations). En l'occurrence, il faut apparemment comprendre, et ceci correspond bien à la manière d'Annon de présenter la chronologie (voir ci-dessus, annexe I, b) que les protagonistes ont convenu de se rencontrer six jours plus tard. La Complainte est la seule à fournir ce détail et à préciser que l'accord s'est fait sans échange d'otages.

75. À comparer à cette formule de la Vie de saint Hugues: «Statuto autem tempore» (VH, 23). 
"Qua ipse [Arnulfus] a terra sua egredi et collocuturis obvenire dignaretur » (RICHER, II, 32, p. 121); "... Ar(nulfus) terra, Uuilelmus aqua in locum destinatum conveniunt.» (ibid., II, 33, p. 122).

«Willelmus, dux praepotentissimus omnium,... profectus est ad Ambianensem usque pagum.» (Dudon, III, 61, p. 206). «Arnulfus vero,... venit super Corbeiae rivulum cum suis omnibus, misitque internuntium ad ducem Willelmum, deprecans contra se venire illum, usque ad Pinchiniacum...» (ibid., III, 62, p. 207).

- On échange des paroles de paix et d'amitié; Arnoul cache bien son jeu:

"Insequenti namque die conglobanti/tantum ore et non corde, ut amici/fatebantur quod loquendum erat illis/simultate latilante.» (Complainte, $\mathrm{X})^{76}$.

"Qui postquam adveniret, et ab amico exceptus esset, de amicicia plurimum, multum etiam de fide proponerent » (RICHER, II, 32, p. 121); «... de amicitia multum, plurimum de fide utrimque servanda collocuti sunt...» (ibid., II, 33, p. 122).

"Tota die pene ducta morosis ambagibus pactaque pace ab utroque principe, Willelmo scilicet fide, atque ab Arnulfo perfido corde cum caeteris principibus..." (Dudon, III, 62-63, p. 207).

"Arnulfo etenim proditoris Iude morem imitato, neniis et ambagibus diutius telam aranee texente, novissime post iurata amicitiarum sacramenta et plurima pacis oscula...» (GND, III, 12, t. 1, p. 92.) ${ }^{77}$.

- Les deux groupes se séparent:

«... post nonnullos sermones a se soluti [...]. Arnulfus reditum simulans, aliquantisper digreditur. Uuilelmus vero ad classem rediit.» (RICHER, II, 33, p. 122) ${ }^{78}$.

76. Pour le vers $\mathrm{n}^{\circ} 2$ (tantum... amici), nous suivons, comme Phillipp Becker, la leçon de Jules Lair, celle-ci correspondant bien à l'idée de dissimulation présente dans le vers $\mathrm{n}^{\circ} 4$ (simultate latitante), ainsi que dans les textes de Dudon et de Guillaume de Jumièges (v. ci-après). Dans la proposition de Philippe Lauer (tantum more et concorde ut amici), les mots tantum more sont dépourvus de sens. Sur l'opposition entre cœur et parole chez Annon, voir ci-dessus, annexe I $b$.

77. Une des interpolations de Guillaume de Jumièges au texte de Dudon. La référence à Judas permet de mieux comprendre le récit de Dudon lorsque celui-ci nous parle des douze compagnons de Guillaume et du baiser d'Arnoul: l'allusion aux apôtres et au baiser de Judas devient évidente. De même, la mention de la nuit tombante et le fait que Guillaume se retrouve seul, séparé de ses compagnons, évoquent l'épisode de l'arrestation de Jésus. Tout se passe comme si, dans la version originale, le "martyre" de Guillaume Longue-Epée était comparé à la Passion du Christ.

78. Le passage «ad classem rediit » de Richer provient évidemment de la même source que le «intrat (que)...classem» de Dudon (v. ci-dessous). 
"Willelmus cum duodecim regreditur, dato osculo Arnulfo, intratque cum remige classem solus, duodecim comitibus altera antecedentibus.» (Dudon, III, 62-63, p. 207).

«ab [sic] invicem dirimuntur.» $(G N D, \text { III, } 12, \text { t. } 1, \text { p. } 92)^{79}$.

- C'est la tombée du jour:

«Occidentem versus sole labescente... » (Complainte, $\mathrm{XI}{ }^{80}$.

«sole ruente ad occasum.» (GND, III, 12, t. 1, p. 92).

- Prétextant qu'Arnoul a oublié de lui dire une chose importante, les conjurés rappellent Guillaume à grands cris :

"... transligati insidiantes: "advenite/ expectate, expectate. / Adhuc latet senioris vos archanum/sibi ipsi atque vobis profuturum" "(Complainte, XI-XII).

"Cumque iam navigaret per pelagus, per coniaturos multo clamore revocaretur, acsi aliquid precipuum oblivione pretermissum auditurus " (RICHER, II, 32, p. 121). «Naviculamque ingressus dum per pelagus navigaret, a coniuratis multo strepitu inclamatus... Illi mox quiddam preotissimum se deferre asserunt quod a domino suo oblivione suppressum fuit.» (ibid., II, 33, p. 122).

"Tunc Eiricus Balzoque et Rotbertus atque Ridulfus perfidi coeperunt subdola reciprocaque voce dicentes Willelmo duci dolose fari: «Domine, Domine, melioris consilii obliti, torque parumper, precamur, navim, quia volumus te paucis. Noster senior... mandat mirabile, cujus oblitus est, tibi.» (Dudon, III, 63-64, p. 207-208).

- Arnoul, soi-disant handicapé par une crise de goutte, attend sans bouger sur l'autre rive:

«oc... expectante prestolando...»(Complainte, XII).

«... dominum cum copiis exspectantem (prestolantem)...» (RICHER, II, 32, p. 122$)^{81}$.

79. "ad invicem ", expression fréquente chez Annon (VA, 17, 19, 25).

80. Nous suivons la lecture de Phillipp Becker, qui corrige Occidente en Occidentem. La formule inchoative sole labescente est à rapprocher de celle qu'Annon utilise dans la Vie de saint Aycadre pour décrire la tombée du jour: vesperascente die $(V A, 58)$.

81. Le mot prestolantem a été rajouté par Richer au-dessus d'exspectantem, sans que ce dernier ait été biffé. Sur l'intérêt de cette addition pour l'identification de la source de Richer, v. ci-dessus, notes 15 et 16 . 
"Noster senior nequit te amplius aggredi, quia podagrae infirmitate scis eum detineri...» (Dudon, III, 63, p. 207) ${ }^{82}$.

- Guillaume, qui commençait à s'éloigner en barque, revient à vive allure:

"Hoc cis intuens expectante prestolando, / quo jubente, festinavit.» (Complainte, $\mathrm{XII)}$.

«Navicula ergo advectus cum paucis...» (RICHER, II, 32, p. 121); «Uuilelmus... proram obvertit. Remigansque ad litus quid vellent sciscitaturus rediit.» (ibid., II, 33, p. 122).

"cum remige... solus...»; "Tunc Willelmus, fide integerrimus, perfidorum precatibus crebrius compulsus, torquet navim celerius...» (Dudon, III, 62-63, p. 207$208)^{83}$.

- Les conjurés ont caché sur eux des armes:

«... occulando obviarunt armillossa/quorum unus caput ejus mucrone...» (Complainte, XIII).

"At illi, sub pellium tegmine jam absconsis quatuor mucronibus...» (Dudon, III, 63 , p. 208) ${ }^{84}$.

- Guillaume, lui, est sans armes:

«Quem videntes punitores... inerme se nuda(s)se... » (Complainte, XIV) ${ }^{85}$.

82. Dudon de Saint-Quentin est le seul à donner cette précision. Comme celle-ci n'apporte pas grand chose à son récit et qu'il l'évoque néanmoins à trois reprises (DuDON, III, 61, 62 et 63, p. 206-208), il est probable qu'il s'agit là d'une information originale, issue de la Vita prima. Ce détail a, en effet, la saveur d'authenticité d'une chose vue. Arrivé en Normandie avant la fin de l'année 942 et nommé abbé de Jumièges l'année suivante, Annon connaissait probablement un ou plusieurs témoins directs de l'assassinat du duc.

83. L'expression torquet... celerius répond au festinavit de la Complainte. Dans les récits d'Annon, le mouvement est toujours rapide. Voir, par exemple, l'évocation du retour de Rome de saint Hugues: ad propria repedebat con festinatione; coepit properare ad domum, VH, 15; occurrit ei obviam, ibid., 16.

84. Noter l'emploi du même terme mucro que dans la Complainte, au lieu de gladius, beaucoup plus courant.

85. Le manuscrit portant: se nuda se, nous proposons la lecture se nudasse, à comparer à un passage des Annales de Saint-Vaast (une des sources probables d'Annon): «... cumque nudassent illum armis suis... » (Annales Vedastini, éd. Bernhard DE Simson, MGH., Scriptores rerum germanicarum in usum scholarum, 12, Hanovre, Hahn, 1909, p. 61). Rappelons toutefois que ce fragment 
«... venitque ad ripam fluminis armorum securus sine suis...» (DUdon, III, 63, p. 208).

- Lorsqu'il rejoint la rive, il est aussitôt assailli et frappé à mort:

«Quem videntes punitores illi duo jugularunt... / Tali modo corpus terre, flatum celo/eum Christo obtulerunt.» (Complainte, XIV) ${ }^{86}$.

«Dux navicula litori appulsa, illos excipit, a quibus etiam mox gladiis eductis interimitur. » (RICHER, II, 33, p. 122).

«... venitque ad ripam fluminis..., cum eis locuturus. At illi,.. quatuor mucronibus celeriter extractis, rabie immanissimi furoris accensi diabolicoque spiritu exagitati, percutiunt et occidunt, heu, dolor! innocentem Willelmum, videntibus cunctis.» (Dudon, III, 63, p. 208) ${ }^{87}$.

- Les deux jeunes qui se trouvaient avec le duc sont blessés, ainsi que le rameur:

"Duobus quoque puberibus qui cum eo inermes aderant, et nauta sauciatis.» (RICHER, II, 33, p. 122) ${ }^{88}$.

- Leur forfait accompli, les assassins rejoignent leur maître et prennent la fuite:

"equis velocibus rapti, ad dominum cum copiis exspectantem (prestolantem) transfugere tempestivius accelerarent » (RICHER, II, 32, p. 122); «Illi... a navicula facinorosi exiliunt, ac post conscium dominum in fugam feruntur. » (ibid., II, 33, p. 122).

lacunaire de la Complainte a donné lieu à des interprétations très diverses: "... sed nuda se...» (Wilhelm Meyer), "... inermes nautas suos» (Philippe Lauer), «... inermem denudarunt» (Phillipp Becker).

86. Nous suggérons de restituer quem à la place du quo du manuscrit. Philippe Lauer et les autres éditeurs optent pour quod.

87. Comme nombre d'auteurs l'ont déjà remarqué avant nous, les mots occidunt... innocentem font écho à la formule innocente interfecto du refrain de la Complainte.

88. Richer est le seul à fournir ces précisions. Cependant, comme l'a conjecturé Philippe Lauer, il pourrait y avoir une relation avec les mots duo et inerme(s) de la Complainte, ceux-ci constituant les fragments d'une strophe perdue, attestée par le seul manuscrit de Florence. Dudon parle, lui aussi, du rameur qui se trouvait dans la barque de Guillaume: «intratque cum remige classem solus " (Dudon, III, 62, p. 207); à rapprocher du mot remigans utilisé un peu plus haut par Richer. 
" hincque, cum domino omnium nequissimo, celeri classe transvecti suoque exercitui annexi, praepete equitatu potiuntur fuga lapsi.» (Dudon, III, 63, p. 208) ${ }^{89}$.

- Le corps de Guillaume gît à terre, mais son âme est déjà montée au ciel:

«... corpus terre, flatum celo/eum Christo obtulerunt.» (Complainte, éd. Lauer, p. 323, XIV).

"... corpus jacuit exanime. Verum anima, in coelum ab angelis deducta, inter choros angelorum inaestimabiliter est collocata.» (Dudon, III, 64, p. 208).

- Inhumation de Guillaume:

«... Sumptumque domini corpus lamentabili obsequio sepulturae deportant.» (RicheR, II, 33, p. 122) ${ }^{90}$.

«Statim vero corpus sacrosanctum feretro velociter impositum et Rotomagensi urbi cum magno ejulatu delatum in ecclesiam B(eate) Mariae genitricis Dei honorifice sepelierunt. »(DUdON, III, 64, p. 208).

89. Les mots suoque exercitui répondent au cum copiis de Richer; noter également, de part et d'autre, l'emploi du même terme dominus pour désigner Arnoul.

90. Richer mentionne souvent le décès des princes, mais il est rare qu'il s'attarde à évoquer le deuil de leurs sujets et leurs funérailles. En l'occurrence, le fait est d'autant plus remarquable que le personnage de Guillaume de Normandie n'est manifestement pas de ceux qui inspirent le plus de sympathie à Richer. Ceci accrédite, une fois de plus, l'hypothèse d'un récit préexistant, repris par le moine de Saint-Rémi. 